STRUCTURAL SCIENCE CRYSTAL ENGINEERING MATERIALS

ISSN 2052-5206

Received 4 January 2021

Accepted 12 March 2021

Edited by R. Černý, University of Geneva, Switzerland

Keywords: quartz; pseudocubic; INA; tilt angle; structure prediction; $\mathrm{GeO}_{2}$.

Supporting information: this article has supporting information at journals.iucr.org/b

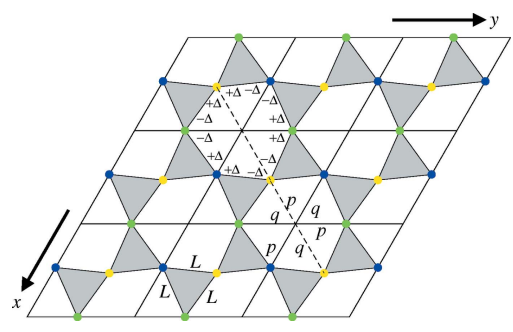

(c) () OPEN O ACCESS

\section{Modelling the structural variation of quartz and germanium dioxide with temperature by means of transformed crystallographic data}

\author{
Maximilian Fricke and Noel W. Thomas* \\ Werkstofftechnik Glas and Keramik, Hochschule Koblenz, Rheinstrasse 56, 56203 Hoehr-Grenzhausen, Germany. \\ *Correspondence e-mail: thomas@hs-koblenz.de
}

The pseudocubic (PC) parameterization of $\mathrm{O}_{4}$ tetrahedra [Reifenberg \& Thomas (2018). Acta Cryst. B74, 165-181] is applied to quartz $\left(\mathrm{SiO}_{2}\right)$ and its structural analogue germanium dioxide $\left(\mathrm{GeO}_{2}\right)$. In $\alpha$-quartz and $\mathrm{GeO}_{2}$, the pseudocubes are defined by three length parameters, $a_{\mathrm{PC}}, b_{\mathrm{PC}}$ and $c_{\mathrm{PC}}$, together with an angle parameter $\alpha_{\mathrm{PC}}$. In $\beta$-quartz, $\alpha_{\mathrm{PC}}$ has a fixed value of $90^{\circ}$. For quartz, the temperature evolution of parameters for the pseudocubes and the silicon ion network is established by reference to the structural refinements of Antao [Acta Cryst. (2016), B72, 249-262]. In $\alpha$-quartz, the curve-fitting employed to express the non-linear temperature dependence of pseudocubic length and Si parameters exploits the model of a first-order Landau phase transition utilized by Grimm \& Dorner [J. Phys. Chem. Solids (1975), 36, 407413]. Since values of tetrahedral tilt angles about $\langle 100\rangle$ axes also result from the pseudocubic transformation, a curve for the observed non-monotonic variation of $\alpha_{\mathrm{PC}}$ with temperature can also be fitted. Reverse transformation of curvederived values of $[\mathrm{Si}+\mathrm{PC}]$ parameters to crystallographic parameters $a, c, x_{\mathrm{Si}}, x_{\mathrm{O}}$, $y_{\mathrm{O}}$ and $z_{\mathrm{O}}$ at interpolated or extrapolated temperatures is demonstrated for $\alpha$ quartz. A reverse transformation to crystallographic parameters $a, c, x_{\mathrm{O}}$ is likewise carried out for $\beta$-quartz. This capability corresponds to a method of structure prediction. Support for the applicability of the approach to $\mathrm{GeO}_{2}$ is provided by analysing the structural refinements of Haines et al. [J. Solid State Chem. (2002), 166, 434-441]. An analysis of trends in tetrahedral distortion and tilt angle in $\alpha$-quartz and $\mathrm{GeO}_{2}$ supports the view that $\mathrm{GeO}_{2}$ is a good model for quartz at high pressure.

\section{Introduction}

Although the $\alpha \leftrightarrow \beta$ quartz inversion has been an issue of scientific investigation for some 130 years (Dolino, 1990), a strong stimulus to review current modelling methods for its crystal structures has been provided by the work of Antao (2016). By using synchrotron powder X-ray diffraction coupled with Rietveld structure refinements, she extended the range of structural data well into the temperature range of stability of $\beta$-quartz and provided a set of structural data for $\alpha$ and $\beta$-quartz with a fine temperature mesh. A total of 67 new structural refinements resulted from her work, 42 for $\alpha$-quartz and 25 for $\beta$-quartz, thereby providing an extensive dataset for structural analysis.

The foundation of several structural modelling studies of quartz and its homeotypes was laid by Grimm \& Dorner (1975), who identified the tilt angle of $\delta$ of $\mathrm{SiO}_{4}$ tetrahedra about $\langle 100\rangle$ axes in $\alpha$-quartz as the microscopic order parameter in a first-order Landau model of the $\alpha \leftrightarrow \beta$ phase transition. Parameter $\delta_{0}$ in equation (1) corresponds to the jump in tilt angle at the transition temperature $T_{0}$, with $T_{\mathrm{c}}$ a scaling parameter. 


$$
\delta^{2}=\frac{2}{3} \delta_{0}^{2}\left[1+\left(1-\frac{3}{4}\left(\frac{T-T_{\mathrm{c}}}{T_{0}-T_{\mathrm{c}}}\right)\right)^{1 / 2}\right]
$$

Grimm and Dorner (1975) assumed regular tetrahedra as a starting point for fitting equation (1) to values of $\delta$ derived from crystallographic data. This resulted in the values $\delta_{0}=$ $7.3^{\circ}, T_{0}=846 \mathrm{~K}$ and $T_{0}-T_{\mathrm{c}}=10 \mathrm{~K}$. They noted that the accuracy of the crystallographic data then available was insufficient to test the validity of equation (1), further that 'a direct measurement of the tilt angle analogous to the case of $\mathrm{SrTiO}_{3}$ would be desirable.'

This notwithstanding, equation (1) has been widely adopted in subsequent studies of the temperature dependence of the structures of $\alpha$-quartz and its homeotypes. This can be attributed to the greater suitability of $\delta$ or $\delta^{2}$ compared to direct temperature as an independent variable when describing the temperature dependence of structural parameters such as the $\mathrm{Si}-\mathrm{O}-\mathrm{Si}$ angle by means of low-order polynomials. It is appropriate, therefore, to regard $\delta$, also denoted $Q$ in later studies, as a temperature-derived tilt angle, irrespective of the degree of agreement with a structurally derived tilt angle.

Carpenter et al. (1998) adopted this approach to derive quadratic relationships between spontaneous strains $e_{1}$ and $e_{3}$ with $Q^{2}$. They also utilized the structural data of Kihara (1990) for $\alpha$-quartz to reveal a linear relationship between the mean $\mathrm{Si}-\mathrm{O}$ bond length and the square of the tilt angle. By virtue of her extensive structural dataset, Antao (2016) has further shown that strain parameters $e_{1}, e_{3},(c / a)$ and volume strain $V_{\mathrm{s}}$ vary linearly with $Q^{2}$ for $\alpha$-quartz She also proposed linear relationships between atomic parameters $z_{\mathrm{O}}$ and $x_{\mathrm{Si}}$ with $Q$. Mean $\mathrm{Si}-\mathrm{Si}$ distances and $\mathrm{Si}-\mathrm{O}-\mathrm{Si}$ angles were also shown to vary systematically with $Q$. In both cases, tilt angle was calculated according to the method of Grimm \& Dorner (1975) assuming regular tetrahedra.

The structural refinements of Antao (2016) refer to space group $P 3_{2} 21$ (No. 154) for $\alpha$-quartz and space group $P 6_{2} 22$ (No. 180) for $\beta$-quartz. The coordinates for $\alpha$-quartz correspond to the $z(+)$-setting (Donnay \& Le Page, 1978) ${ }^{\mathbf{1}}$. When cooling right-handed $\beta$-quartz (space group $P 6_{2} 22$ ), formation either of an $\alpha_{1}$ or an $\alpha_{2}$ trigonal structure depends on the sense of the tetrahedral tilting. ${ }^{2}$ These are in space groups $P 3_{2} 21$ and $P 3_{1} 21$, respectively.

Ever since the early crystal-chemical treatments of quartz, the view has dominated that the $\mathrm{SiO}_{4}$ tetrahedra deviate insignificantly from perfect regularity. Megaw (1973a) states this clearly: 'We have already recognized the importance of a regular (or nearly regular) tetrahedron as a structure-building unit.' In the seminal work of Grimm \& Dorner (1975) in relating tetrahedral tilt angle to the Landau order parameter in equation (1), the assumption of regular tetrahedra was

\footnotetext{
${ }^{1}$ In order to generate the coordinates of Donnay \& Le Page (1978), it would be necessary to shift the unit cell origin by $\left[0,0,-\frac{1}{3}\right]$ from the origin in International Tables of X-ray Crystallography (Hahn, 1995). The latter was adopted by Antao (2016).

${ }^{2}$ The $\alpha_{1}$ and $\alpha_{2}$ structures are Dauphiné twins related by a $180^{\circ}$ rotation about the threefold axis (Antao, 2016).
}

maintained as an expedient. Taylor (1984) explicitly called this assumption into question, to quote from his abstract: 'Tilting models of framework compounds are critically examined and their failure to match the observed structural behaviour is attributed to changes in tetrahedral distortion. For quartz it appears that during compression the change in tetrahedral distortion is virtually all angular $(\mathrm{O}-\mathrm{Si}-\mathrm{O}$ angles), whereas during thermal expansion the change in distortion is in the $\mathrm{Si}-\mathrm{O}$ distances. Such behaviour may typify the behaviour of many other framework compounds but the structural data needed to establish this are lacking.'

The current availability of high-quality structural data for quartz following the work of Antao (2016) now supersedes the final remark of Taylor for this framework compound. Furthermore, a new approach for quantifying the distortions of $\mathrm{O}_{4}$ tetrahedra has recently been proposed by Reifenberg \& Thomas (2018). In the latter work, the pressure variation of the structure of the coesite polymorph of $\mathrm{SiO}_{2}$ was taken as a basis for defining a general procedure known as a pseudocubic transformation. Just as it is possible to generate a regular tetrahedron from a cube by taking two diagonally related corners of each cube face, the reverse procedure also holds: a regular tetrahedron will generate a regular cube, whereas a distorted tetrahedron will generate a distorted cube known as a pseudocube (Fig. 1). Such a pseudocube is, in general, characterized by six parameters, $a_{\mathrm{PC}}, b_{\mathrm{PC}}, c_{\mathrm{PC}}, \alpha_{\mathrm{PC}}, \beta_{\mathrm{PC}}$ and $\gamma_{\mathrm{PC}}$ (Fig. 1), as for a triclinic unit cell. As shown in Fig. 1(a), the shape of a generalized tetrahedron is also defined by six parameters. It follows that all volumes and types of distortion of tetrahedral $\mathrm{O}_{4}$ cages can be quantitatively modelled by pseudocubic transformations.

Whereas the distorted $\mathrm{O}_{4}$ tetrahedra in coesite result in six independent pseudocubic parameters, the twofold symmetry axes through their centres-of-coordinates in $\alpha$-quartz dictate that two of the pseudocubic angles are equal to $90^{\circ}$.

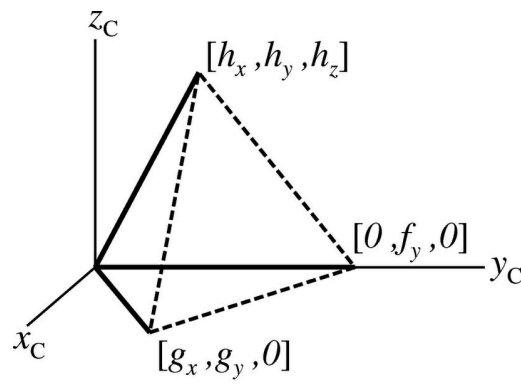

(a)

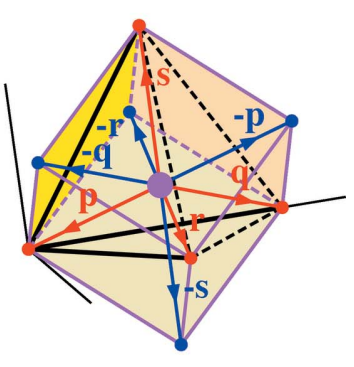

(b)
Figure 1

(a) The form of a generalized tetrahedron is defined by six parameters: $f_{y}$, $g_{x}, g_{y}, h_{x}, h_{y}$ and $h_{z}$. These correspond to the non-zero components of its three bounding vectors in Cartesian coordinates. $(b)$ A pseudocube is formed from the tetrahedron by inverting the four vectors from its centreof-coordinates (large light purple circle) to oxygen ions, i.e. $\mathbf{p}, \mathbf{q}, \mathbf{r}$ and $\mathbf{s}$, to form vectors $-\mathbf{p},-\mathbf{q},-\mathbf{r}$ and $-\mathbf{s}$. Small red circles: pseudocube vertices occupied by oxygen ions; small blue circles: vacant pseudocube vertices (taken from Reifenberg \& Thomas, 2018). 


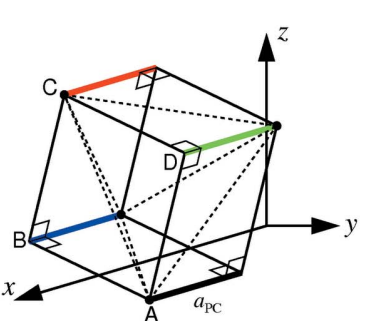

(a)

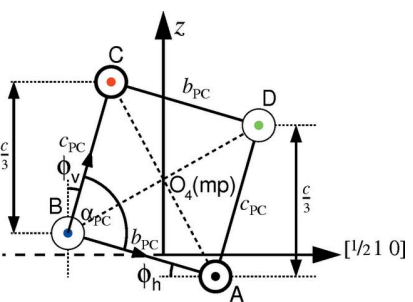

(b)
Figure 2

(a) The $\mathrm{O}_{4}$ tetrahedron corresponding to the silicon ion at $x, 0, \frac{2}{3}$ (as in space group $P_{3} 21$ ) and its corresponding pseudocube. Length $a_{\mathrm{PC}}$ is shown. (b) The pseudocube shown in projection perpendicular to the $x$ axis $\left[\mathrm{O}_{4}(\mathrm{mp})\right.$ : centre-of-coordinates of $\mathrm{O}_{4}$ tetrahedron and associated pseudocube; $\phi_{\mathrm{h}}, \phi_{\mathrm{v}}$ : horizontally and vertically defined pseudocubic tilt angles]. (Modifed from an original figure given by Reifenberg \& Thomas, 2018.)

This is shown in Fig. 2(a), in which pseudocubic axes $a_{\mathrm{PC}}$ are oriented parallel to the twofold axes. The face-on view of the pseudocube along the $x$-axis in Fig. 2(b) shows a parallelogram with twofold symmetry and internal angle $\alpha_{\mathrm{PC}}$.

A secondary result of the pseudocubic transformation is that it allows angles $\phi_{\mathrm{v}}$ and $\phi_{\mathrm{h}}$ to be defined as direct indicators of tetrahedral tilt angle $\phi$ about the [100] axis, and more generally $\langle 100\rangle$ axes: a tetrahedral rotation by this angle also leads to a rotation $\phi$ of its pseudocube about the same axis. However, unlike the tetrahedral edge vectors, the edge vectors of the pseudocube are aligned with the crystal axes. Angle $\phi_{\mathrm{v}}$ is defined as the angle between pseudocubic axis $c_{\mathrm{PC}}$ and crystal axis $z$ and angle $\phi_{\mathrm{h}}$ as the angle between pseudocubic axis $b_{\mathrm{PC}}$ and its projection in the crystal $x y$ plane. Owing to small deviations of pseudocubic angle $\alpha_{\mathrm{PC}}$ from $90^{\circ}, \phi_{\mathrm{h}}$ and $\phi_{\mathrm{v}}$ are not exactly equal to each other. Nevertheless, a method is now provided for measuring the tetrahedral tilt angle directly, as sought by Grimm \& Dorner (1975). The method does not require any approximations or abstract geometrical reference points other than the crystal axes. In the current work, the dependence of $\phi_{\mathrm{h}}, \phi_{\mathrm{v}}$ and mean tilt angle $\phi=\left(\phi_{\mathrm{h}}+\phi_{\mathrm{v}}\right) / 2$ on temperature-derived tilt angle $\delta$ (or equivalently $Q$ ) are examined, thereby revealing the extent to which equation (1) holds for $\alpha$-quartz.

The significance of a direct measurement of tilt angle may be made clear by comparing the completely general pseudocubic method with alternative structural approaches advocated by Megaw (1973b) and Grimm \& Dorner (1975) for quartz, as well as the method of Haines et al. (2003) adopted for the quartz homeotype $\mathrm{FePO}_{4}$. Megaw adopted as a basis an idealized tetrahedron of orthorhombic symmetry, as in $\beta$-quartz, and maintained this form as an approximation in $\alpha$-quartz. This approach is equivalent to allowing a pseudocube with unequal edge lengths but with angle $\alpha_{\mathrm{PC}}$ fixed at $90^{\circ}$. The method of Grimm and Dorner is more restrictive, as it amounts to assuming a regular cube as the pseudocubic form. Haines et al., by comparison, examined the deviations in orientation of tetrahedral edges PR and QS from $\pm 45^{\circ}$ (Fig. 3 ).

Fig. 3 shows the alternative senses of tilt in space group $P 3_{2} 21$ for $\alpha$-quartz and in its enantiomorphic space group
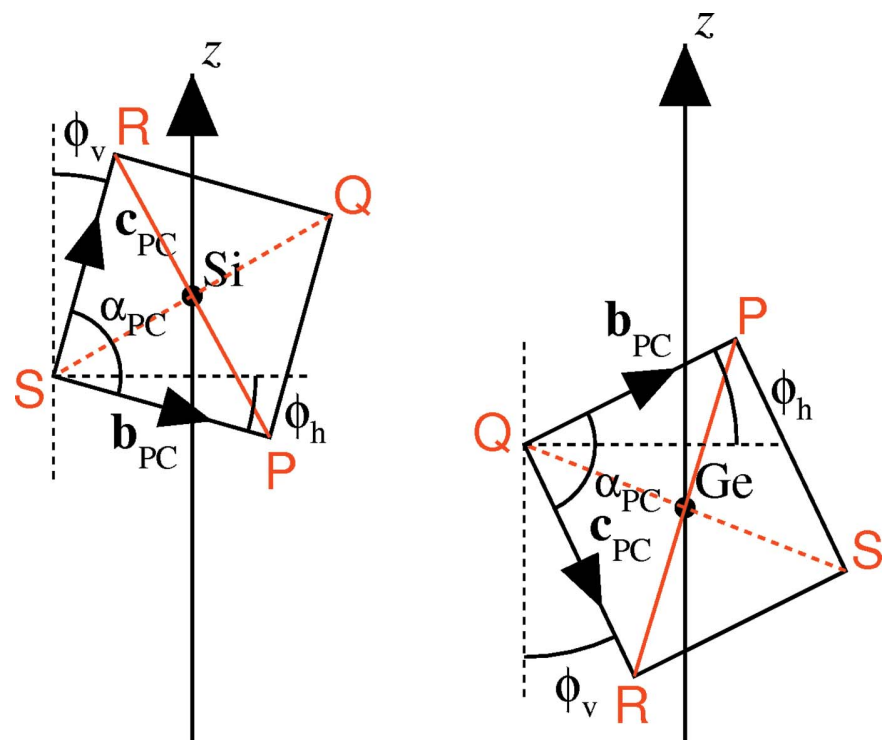

Figure 3

Oxygen ion pseudocubes (left) in $\alpha$-quartz (space group $P 3_{2} 21$ ) and (right) in $\mathrm{GeO}_{2}$ (space group $P 3_{1} 21$ ) viewed along the crystallographic $x$ axis with the $z$-axis vertical. Vectors $\boldsymbol{b}_{\mathrm{PC}}$ and $\boldsymbol{c}_{\mathrm{PC}}$ are the two axes of the pseudocube that enclose angle $\alpha_{\mathrm{PC}}$. Angles $\phi_{\mathrm{h}}$ and $\phi_{\mathrm{v}}$ are tilt angles with horizontal and vertical reference directions. Lines PR and QS are tetrahedral edges (solid lines: at front; dashed lines: at rear).

$P 3_{1} 21$, in which the 16 structures of $\mathrm{GeO}_{2}$ between 294 and $1344 \mathrm{~K}$ to be examined here were set (Haines et al., 2002). ${ }^{\mathbf{3}}$

In addition to investigating the validity of equation (1) in describing the temperature variation of tilt angle as determined by the pseudocubic method, an important further aim of this work is to exploit the pseudocubic transformation for the purpose of structure prediction at temperatures outside the ranges of experimental investigation of Antao (2016) and Haines et al. (2002). Since the pseudocubes only relate to the oxygen ions, the silicon or germanium ions are treated in a separate cationic network. This is consistent with the general methodology of ionic network analysis (INA) (Thomas, 2017). In Fig. 4, the positions of the silicon ions along the screw axes in $\alpha$-quartz have been collapsed on to the $x y$ plane, in order to form a two-dimensional framework defined by parameters $L$ and $\Delta . \Delta$ is equal to zero in the higher-symmetry $\beta$-structure.

The crystal structures of $\alpha$-quartz and $\mathrm{GeO}_{2}$ are defined by two unit-cell and four positional parameters, i.e. $a, c, x_{\mathrm{Si}}, x_{\mathrm{O}}, y_{\mathrm{O}}$ and $z_{\mathrm{O}}$, which are known collectively as six degrees of freedom (d.o.f.). In $\beta$-quartz, by comparison, there are three degrees of freedom ${ }^{4}$, i.e. $a, c$ and $x_{\mathrm{O}}$. The question arises as to how many independent transformed parameters are required to define the $\mathrm{O}_{4}$ pseudocubes and silicon ion networks in the two quartz modifications. For $\alpha$-quartz, six independent parameters are

\footnotetext{
${ }^{3} \mathrm{GeO}_{2}$ was set in the $r(+)$ setting according to Donnay and Le Page (1978). In order to generate the coordinates of Donnay and Le Page, it would be necessary to shift the unit cell origin by $\left[0,0,-\frac{1}{3}\right]$ from the origin in International Tables of X-ray Crystallography (Hahn, 1995). The latter was used by Haines et al. (2002).

${ }^{4}$ Use of the concept of structural degrees of freedom has been made freely in earlier work (Thomas, 2017; Reifenberg \& Thomas, 2018).
} 


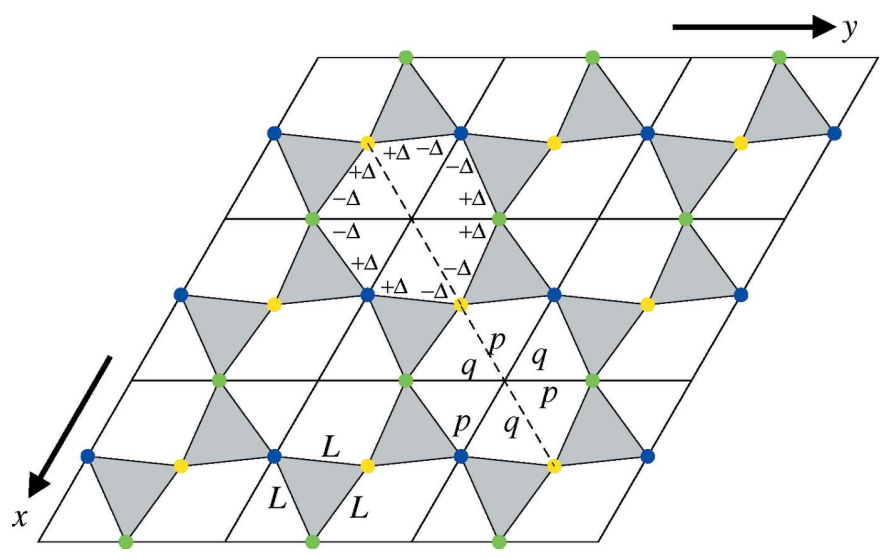

Figure 4

Silicon ions (blue, green and yellow circles) of $\alpha$-quartz in $x y$-projection in space group $P 3_{2} 21$ (blue: $z=\frac{2}{3}$; green: $z=\frac{1}{3}$; yellow: $z=0$ ). These form a $2 \mathrm{D}$ framework characterized by two parameters, $L$ (equilateral triangle sidelength) and $\Delta$ (deviation of angle from $60^{\circ}$ in constitutive triangle of hexagonal void). Lengths $p$ and $q$ show the unequal radii of the hexagonal voids, with $p<q$ (taken from Figure 12 of Reifenberg and Thomas, 2018).

required, i.e. $a_{\mathrm{PC}}, b_{\mathrm{PC}}, c_{\mathrm{PC}}, \alpha_{\mathrm{PC}}, L$ and $\Delta$. These match exactly the six d.o.f. of the structure. In $\beta$-quartz, just three independent parameters are required, although the pseudocubes and silicon ion network deliver four: $a_{\mathrm{PC}}, b_{\mathrm{PC}}, c_{\mathrm{PC}}$ and $L$. This disparity is resolved by noting that parameters $a_{\mathrm{PC}}$ and $b_{\mathrm{PC}}$ are interdependent. ${ }^{5}$ It should also be noted that the tetrahedral tilt angle in $\alpha$-quartz, $\phi$, is not a transformed parameter in this sense: if the six crystal structural parameters or alternatively the six independent transformed parameters are known, the value of $\phi$ follows by calculation.

This article is structured as follows. In $\$ 2$, analytical expressions are given for the values of transformed parameters $a_{\mathrm{PC}}, b_{\mathrm{PC}}, c_{\mathrm{PC}}, \alpha_{\mathrm{PC}}, L$ and $\Delta$, henceforth denoted [Si+PC] or $[\mathrm{Ge}+\mathrm{PC}]$, in terms of crystal structural parameters $a, c, x_{\mathrm{Si}}$ or $x_{\mathrm{Ge}}, x_{\mathrm{O}}, y_{\mathrm{O}}$ and $z_{\mathrm{O}}$. An expression is also given for tilt angles $\phi_{\mathrm{v}}$ and $\phi_{\mathrm{h}}$ in terms of crystal structural parameters. In $\S 3.1$, the transformed parameters calculated for $\alpha$-quartz are summarized by reference to Table $\mathrm{S} 1$ in $\S 4$ of the supporting information. Sections $\$ 3.2$ to $\$ 3.4$ refer to $\alpha$-quartz: the temperature variation of the three tilt angles $\phi_{\mathrm{v}}$ and $\phi_{\mathrm{h}}$ and mean tilt angle $\phi=\left(\phi_{\mathrm{h}}+\phi_{\mathrm{v}}\right) / 2$ is compared to the temperaturederived value of tilt angle according to equation (1) in $\S 3.2$. This equation is subsequently exploited as a baseline curve for a quantitative description of the variation of the three tilt angles with temperature. In $\S 3.3$, curves are derived for the temperature variation of [ $\mathrm{Si}+\mathrm{PC}]$ parameters in $\alpha$-quartz, with their application for the purpose of structure prediction shown in $\$ 3.4$. $\$ 3.5$ deals with $\mathrm{GeO}_{2}$ as a whole, referring to Table $\mathrm{S} 2$ in $\S 4$ of the supporting information. In $\S 3.6, \beta$-quartz is likewise dealt with as a whole, with reference made to Table S3. In $\$ 4.1$ a comparison of the temperature- and pressure-dependent behaviour of $\alpha$-quartz and $\mathrm{GeO}_{2}$ is made, with a discussion of the significance of tetrahedral distortions in framework structures carried out in $\$ 4.2$.

5 This is discussed in $\S S 3$ of the supporting information.
2. Parameterization of the cation frameworks and the $\mathrm{O}_{4}$ tetrahedra in $\alpha$-quartz, $\mathrm{GeO}_{2}$ and $\beta$-quartz structures

The analytical treatment here applies to the three space groups relevant to the experimental data of Antao (2016) and Haines et al. (2002), i.e. $P 3_{2} 21, P 3_{1} 21$ and $P 6_{2} 22$. Although the notation $x_{\mathrm{Si}}$ is used, it is to be understood that this also applies to the $x$-coordinate for germanium in the $\mathrm{GeO}_{2}$ structure. The equations quoted here are derived as follows in the supporting information: $\S S 1$ : cationic network parameters $L$ and $\Delta$ in $\alpha$-quartz and $\mathrm{GeO}_{2} ; \S \mathrm{S} 2$ : PC parameters and tilt angles in $\alpha$-quartz and $\mathrm{GeO}_{2}$; §S3: PC parameters in $\beta$-quartz. These derivations are based on the appropriate space group symmetry, in order to fix the Si or Ge ions in space and to form connected $\mathrm{O}_{4}$ tetrahedral cages.

\subsection{The cationic network}

The transformations from $a$ and $x_{\mathrm{Si}}$ to $L$ and $\Delta$ for $\alpha$-quartz are as follows:

$$
\begin{gathered}
L=a\left(1-3 x_{\mathrm{Si}}+3 x_{\mathrm{Si}}^{2}\right)^{1 / 2}, \\
\Delta=\arccos \left[\frac{1}{2\left(1-3 x_{\mathrm{Si}}+3 x_{\mathrm{Si}}^{2}\right)^{1 / 2}}\right] .
\end{gathered}
$$

In the case of $\beta$-quartz, the value $x_{\mathrm{Si}}=0.5$ leads to the results $L=a / 2$ and $\Delta=0$.

Reverse transformation from $[\mathrm{Si}+\mathrm{PC}]$ to crystal structural parameters proceeds according to equations (4) and (5).

$$
a=2 L \cos \Delta
$$

Quadratic equation (5) follows from equation (1):

$$
3 x_{\mathrm{Si}}^{2}-3 x_{\mathrm{Si}}+1-\frac{L^{2}}{a^{2}}=0
$$

The smaller of the two roots corresponds to the value of $x_{\mathrm{Si}}$.

2.2. Pseudocubic parameters and tilt angles in $a$-quartz and germanium dioxide

The six parameters of the pseudocubes for the $\mathrm{O}_{4}$ tetrahedra may be calculated as follows from unit-cell parameters $a$ and $c$ together with the $x, y$ and $z$ parameters of the oxygen ions:

$$
a_{\mathrm{PC}}=\left|a\left(\frac{3 x_{\mathrm{O}}}{2}-1\right)\right| .
$$

The expression for parameter $b_{\mathrm{PC}}$ depends on whether space group $P 3_{2} 21$ or space group $P 3_{1} 21$ applies, as for $\alpha$-quartz and $\mathrm{GeO}_{2}$, respectively.

$$
b_{\mathrm{PC}}=\left[\frac{3}{4} a^{2} x_{\mathrm{O}}^{2}+c^{2}\left(2 z_{\mathrm{O}}-\frac{5}{3}\right)^{2}\right]^{1 / 2}
$$

[for space group $P 3_{2} 21$ ], 


$$
b_{\mathrm{PC}}=\left[\frac{3}{4} a^{2} x_{\mathrm{O}}^{2}+c^{2}\left(2 z_{\mathrm{O}}-\frac{1}{3}\right)^{2}\right]^{1 / 2}
$$

[for space group $P 3_{1} 21$,

$$
c_{\mathrm{PC}}=\left[\frac{3 a^{2}}{4}\left(-x_{\mathrm{O}}+2 y_{\mathrm{O}}\right)^{2}+\frac{c^{2}}{9}\right]^{1 / 2}
$$

The results for parameter $\alpha_{\mathrm{PC}}$ are likewise dependent on the space group that applies.

$$
\alpha_{\mathrm{PC}}=\arccos \left[\frac{\frac{1}{3} c^{2}\left(2 z_{\mathrm{O}}-\frac{5}{3}\right)-\frac{3}{4} a^{2}\left(x_{\mathrm{O}}^{2}-2 x_{\mathrm{O}} y_{\mathrm{O}}\right)}{b_{\mathrm{PC}} c_{\mathrm{PC}}}\right]
$$

[for space group $P 3_{2} 21$ ],

$$
\alpha_{\mathrm{PC}}=\arccos \left[\frac{-\frac{1}{3} c^{2}\left(2 z_{\mathrm{O}}-\frac{1}{3}\right)-\frac{3}{4} a^{2}\left(x_{\mathrm{O}}^{2}-2 x_{\mathrm{O}} y_{\mathrm{O}}\right)}{b_{\mathrm{PC}} c_{\mathrm{PC}}}\right]
$$

[for space group $P 3_{1} 21$ ]

$$
\beta_{\mathrm{PC}}=\gamma_{\mathrm{PC}}=90^{\circ}
$$

In Figs. 3(a) and 3(b), tilt angles $\phi_{\mathrm{v}}$ and $\phi_{\mathrm{h}}$ are shown for tetrahedra with cations at $x_{\mathrm{Si}}, 0, \frac{2}{3}$ and $x_{\mathrm{Ge}}, 0, \frac{1}{3}$ in $\alpha$-quartz and $\mathrm{GeO}_{2}$, respectively. In both cases,

$$
\phi_{\mathrm{v}}=\arccos \left(\frac{c}{3 c_{\mathrm{PC}}}\right)
$$

and

$$
\phi_{\mathrm{h}}=\arccos \left[\frac{(3)^{1 / 2} a x_{\mathrm{O}}}{2 b_{\mathrm{PC}}}\right] .
$$

Calculation of the mean tilt-angle,

$$
\phi=\frac{\left(\phi_{\mathrm{v}}+\phi_{\mathrm{h}}\right)}{2},
$$

is straightforward. From the geometry in Fig. 3, it follows that

$$
\alpha_{\mathrm{PC}}=90^{\circ}-\left(\phi_{\mathrm{v}}-\phi_{\mathrm{h}}\right) \text {. }
$$

Equation (16) represents an alternative to equations (10) and (11) for calculating the pseudocubic angle $\alpha_{\mathrm{PC}}$. It also reveals how deviations of the pseudocubic angle from $90^{\circ}$ result from differences in the values of tilt-angles $\phi_{\mathrm{v}}$ and $\phi_{\mathrm{h}}$.

The INA method demands that reverse transformations from pseudocubic to crystal structural parameters can take place. In this connection, equations (4) and (5) relating to the cationic network enable this for cell parameter $a$ and cation parameter $x_{\mathrm{Si}}$. The remaining four parameters, i.e. c, $x_{\mathrm{O}}, y_{\mathrm{O}}, z_{\mathrm{O}}$, may be calculated as follows from the pseudocubic parameters. Parameter $x_{\mathrm{O}}$ is derived from $a_{\mathrm{PC}}$ via equation (6). Parameters $c, y_{\mathrm{O}}$ and $z_{\mathrm{O}}$ are derived from the values of $b_{\mathrm{PC}}, c_{\mathrm{PC}}$ and $\alpha_{\mathrm{PC}}$ by finding self-consistent solutions of equations (7) to (11) using numerical methods. These reverse transformations are carried out in $\$ 3.4$ for $\alpha$-quartz.

\subsection{Pseudocubic parameters in $\beta$-quartz}

The six parameters of the pseudocubes for the $\mathrm{O}_{4}$ tetrahedra in $\beta$-quartz may similarly be calculated analytically from unit-cell parameters $a$ and $c$ together with the $x_{\mathrm{O}}$ parameter of the oxygen ions ${ }^{6}$ :

$$
\begin{gathered}
a_{\mathrm{PC}}=(3)^{1 / 2} a x_{\mathrm{O}}, \\
b_{\mathrm{PC}}=\left|a\left(3 x_{\mathrm{O}}-1\right)\right|, \\
c_{\mathrm{PC}}=\frac{c}{3}, \\
\alpha_{\mathrm{PC}}=\beta_{\mathrm{PC}}=\gamma_{\mathrm{PC}}=90^{\circ} .
\end{gathered}
$$

The lengths of pseudocubic axes $a_{\mathrm{PC}}$ and $b_{\mathrm{PC}}$ are interdependent, since both are determined by parameters $a$ and $x_{\mathrm{O}}$. Parameter $L$ in the cation network is equal to twice the unitcell parameter $a$, and the pseudocubes yield values for $x_{0}$ and $c$ by reverse transformation. These transformations are carried out in $\$ 3.6$.

3. The temperature variation of $[\mathrm{Si}+\mathrm{PC}]$ parameters for quartz and $[\mathrm{Ge}+\mathrm{PC}]$ parameters for germanium dioxide

\subsection{Parameters calculated for $\alpha$-quartz}

[Si+PC] parameters calculated for $\alpha$-quartz from the data of Antao (2016) are listed in Table S1. Also listed are the volumes of the unit cell $\left(V_{\mathrm{UC}}\right)$, tetrahedral volumes $\left(V_{\text {tetra }}\right)$, the ratios of the volume occupied by tetrahedra to the unit-cell volume $\left(3 V_{\text {tetra }} / V_{\mathrm{UC}}\right)$, the length-based tetrahedral distortion parameters $\left(\lambda_{\mathrm{PC}}\right)$ [equation (21); Reifenberg \& Thomas, 2018], together with tilt angles $\phi_{\mathrm{v}}$ and $\phi_{\mathrm{h}}$.

$$
\lambda_{\mathrm{PC}}=\frac{\left|a_{\mathrm{PC}}-L_{0, \mathrm{PC}}\right|+\left|b_{\mathrm{PC}}-L_{0, \mathrm{PC}}\right|+\left|c_{\mathrm{PC}}-L_{0, \mathrm{PC}}\right|}{3 L_{0, \mathrm{PC}}}
$$

with $L_{0, \mathrm{PC}}=\left(a_{\mathrm{PC}}+b_{\mathrm{PC}}+c_{\mathrm{PC}}\right) / 3$.

3.2. Curve-fitting for the temperature variation of tilt angles in $a$-quartz

The correlation of values of order parameter $\delta$ calculated from equation (1) using the parameters of Grimm \& Dorner (1975) with values of $\phi_{v}, \phi_{\mathrm{h}}$ and $\phi$ calculated directly from the structural refinements of Antao (2016) via equations (13) to (15) is shown in Fig. 5.

It is observed that the correlation between the black curve and the other three curves is only qualitative. This indicates that, although the predominant contribution to the microscopic Landau order parameter is made by tetrahedral tilting, there will also be a small contribution to this from tetrahedral distortion.

Fitting of the curves linking experimental points for $\phi_{\mathrm{v}}, \phi_{\mathrm{h}}$ and $\phi_{\mathrm{m}}$ was carried out by expressing these three parameters as a function of $\delta$, using polynomials of order 3 . The fitting coefficients are listed in Table 1.

${ }^{6}$ Values of $x_{0}$ in equations (17) and (18) are in accordance with the notation in International Tables for Crystallography (Hahn, 1995) and equal to one half of the values of $\mathrm{O}_{x}$ quoted by Antao (2016). 


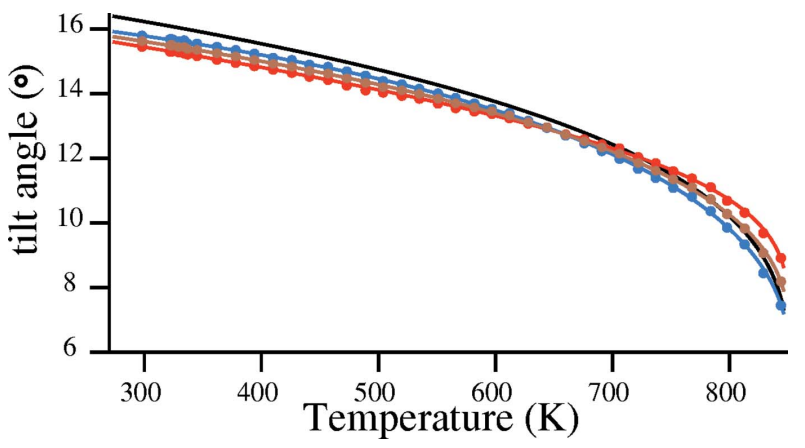

Figure 5

Comparison of the order parameter $\delta$ (black curve) with structurally derived values of tilt angles, $\phi_{\mathrm{v}}, \phi_{\mathrm{h}}$ and $\phi$ with points and curves in red, blue and brown, respectively. Temperature range: $273-846 \mathrm{~K}$.

Table 1

Fitting coefficients for tilt angles $\phi_{\mathrm{v}}, \phi_{\mathrm{h}}$ and $\phi$ in $\alpha$-quartz.

\begin{tabular}{lccc}
\hline & $\phi_{\mathrm{v}}\left({ }^{\circ}\right)$ & \multicolumn{1}{c}{$\phi_{\mathrm{h}}\left({ }^{\circ}\right)$} & $\phi\left(^{\circ}\right)$ \\
\hline$a_{0}$ & $1.19197 \times 10^{0}$ & $6.32541 \times 10^{0}$ & $3.77495 \times 10^{0}$ \\
$a_{1}$ & $1.38790 \times 10^{0}$ & $-7.60768 \times 10^{-1}$ & $3.08692 \times 10^{-1}$ \\
$a_{2}$ & $-6.68003 \times 10^{-2}$ & $1.50911 \times 10^{-1}$ & $4.21756 \times 10^{-2}$ \\
$a_{3}$ & $2.18006 \times 10^{-3}$ & $-4.19733 \times 10^{-3}$ & $-1.00113 \times 10^{-3}$ \\
r.m.s.d. $\left({ }^{\circ}\right)$ & $3.82 \times 10^{-2}$ & $6.16 \times 10^{-8}$ & $1.60 \times 10^{-8}$ \\
\hline
\end{tabular}

\subsection{Curve fitting for the temperature variation of [Si+PC]-} parameters in $\alpha$-quartz

Values of parameters $L, \Delta, a_{\mathrm{PC}}, b_{\mathrm{PC}}, c_{\mathrm{PC}}$ and $\alpha_{\mathrm{PC}}$ from Table S1 for temperatures between 298 and $844 \mathrm{~K}$ are plotted as points with associated error bars in Fig. 6.

The method adopted for fitting the curves was consistent with the work of other authors (Grimm \& Dorner, 1975; Carpenter et al., 1998; Antao, 2016), in that the order parameter $\delta$ generated by equation (1) was adopted as the independent variable. The fitting coefficients listed in Table 2 relate to the reduced order parameter $\delta^{\prime}$ defined in equation (22)

$$
\delta^{\prime}=\frac{\left[\delta(T)-\delta_{0}\right]}{\left[\delta(273 \mathrm{~K})-\delta_{0}\right]} .
$$

Here $\delta_{0}$ is the parameter of Grimm \& Dorner (1975), which is equal to $7.3^{\circ}$. This is their tilt angle at the temperature $T_{0}$, which is equal to $846 \mathrm{~K}$. Parameter $\delta(273 \mathrm{~K})$ is calculated by equation (1) to be $16.40^{\circ} . \delta(T)$ is the tilt angle calculated from equation (1) for a temperature lying between 273 and $846 \mathrm{~K}$. Therefore equation (22) delivers a parameter between 0 and 1 for decreasing temperatures between 846 and $273 \mathrm{~K}$, respectively. The fitted curves are shown in Fig. 6. It should be noted

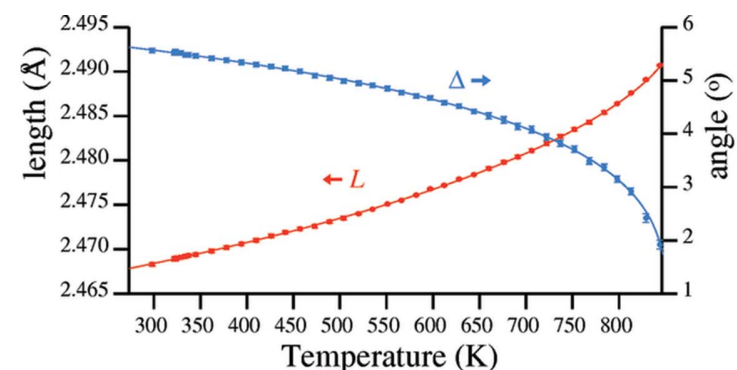

(a)

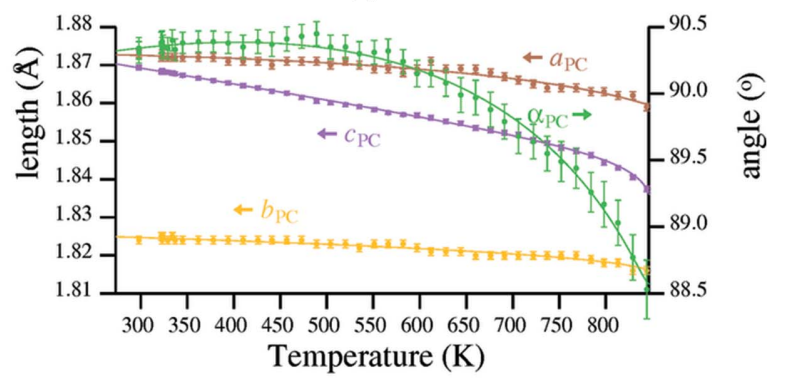

(b)

Figure 6

(a) Silicon framework parameters $L$ and $\Delta$; (b) oxygen pseudocube parameters $a_{\mathrm{PC}}, b_{\mathrm{PC}}, c_{\mathrm{PC}}$ and $\alpha_{\mathrm{PC}}$.

that the use of polynomial coefficients allows parameters $L, \Delta$, $a_{\mathrm{PC}}, b_{\mathrm{PC}}$ and $c_{\mathrm{PC}}$ to vary independently of one another, even though a single Landau order parameter calculated from temperature according to equation (1) is at the core of the fitting method. As a formal contribution to the method, the Landau function provides a more linear baseline that enables the fitting of low-order polynomials. If the five parameter values were fitted directly to reduced temperature, a higher order would be required in order to accommodate the significant non-linearity in the parameter-variation in the region of the phase transition, i.e. at $T \leq T_{c}$. However, such a step would also introduce undesirable short-range artefacts in the fitted curves of questionable physical basis.

The curve fitted for parameter $\alpha_{\mathrm{PC}}$ was calculated from equation (17) utilizing values for tilt angles $\phi_{\mathrm{v}}$ and $\phi_{\mathrm{h}}$ calculated from the coefficients in Table 1 and shown in Fig. 5.

\subsection{Structural prediction for $\alpha$-quartz via the INA method}

The fine temperature-mesh adopted by Antao (2016) means that there is more to be gained by calculating crystal structures outside the range of $298-844 \mathrm{~K}$ than by calculating structures at intermediate temperatures. Therefore four of the temperatures chosen for Table 3, $273 \mathrm{~K}, 283 \mathrm{~K}, 293 \mathrm{~K}$ and $846 \mathrm{~K}$ lie outside this range. A large separation in tempera-

Table 2

Fitting coefficients for parameters $L, \Delta, a_{\mathrm{PC}}, b_{\mathrm{PC}}$ and $c_{\mathrm{PC}}$ in $\alpha$-quartz [equation (A1.2)].

\begin{tabular}{lllrr}
\hline Parameter & $a_{0}$ & \multicolumn{1}{c}{$a_{1}$} & \multicolumn{1}{c}{$a_{2}$} & \multicolumn{1}{c}{$a_{3}$} \\
\hline$L(\AA)$ & $2.49095 \times 10^{0}$ & $-6.87965 \times 10^{-3}$ & $-2.41458 \times 10^{-2}$ & $7.89793 \times 10^{-3}$ \\
$\Delta\left({ }^{\circ}\right)$ & $1.73590 \times 10^{0}$ & $4.47954 \times 10^{0}$ & $-4.07902 \times 10^{-1}$ & $-1.80983 \times 10^{-1}$ \\
$a_{\mathrm{PC}}(\AA)$ & $1.85951 \times 10^{0}$ & $2.23242 \times 10^{-3}$ & $2.64983 \times 10^{-2}$ & $-1.55218 \times 10^{-2}$ \\
$b_{\mathrm{PC}}(\AA)$ & $1.81594 \times 10^{0}$ & $6.10459 \times 10^{-3}$ & $3.41215 \times 10^{-3}$ & $-1.14 \times 10^{-5}$ \\
$c_{\mathrm{PC}}(\AA)$ & $1.83589 \times 10^{0}$ & $3.27551 \times 10^{-2}$ & $-2.31572 \times 10^{-2}$ & $6.98 \times 10^{-4}$ \\
\hline
\end{tabular}


Table 3

[Si+PC]-parameters for $\alpha$-quartz at ten temperatures calculated from the INA curves in Fig. 6 and associated polynomial coefficients in Tables 1 and 2.

Corresponding, calculated crystallographic parameters are listed below the horizontal rule.

\begin{tabular}{|c|c|c|c|c|c|c|c|c|c|c|c|}
\hline$T(\mathrm{~K})$ & 273 & 283 & 293 & 300 & 400 & 500 & 600 & 700 & 800 & 846 & Equation \\
\hline$\delta\left({ }^{\circ}\right)$ & 16.404 & 16.342 & 16.279 & 16.235 & 15.549 & 14.744 & 13.753 & 12.427 & 10.191 & 7.300 & (1) \\
\hline$\phi_{\mathrm{v}}\left({ }^{\circ}\right)$ & 15.607 & 15.548 & 15.488 & 15.446 & 14.817 & 14.121 & 13.316 & 12.307 & 10.706 & 8.612 & $(A 1.1)$ \\
\hline$\phi_{\mathrm{h}}\left({ }^{\circ}\right)$ & 15.927 & 15.877 & 15.826 & 15.789 & 15.203 & 14.461 & 13.488 & 12.121 & 9.803 & 7.181 & $(A 1.1)$ \\
\hline$\alpha_{\mathrm{PC}}\left({ }^{\circ}\right)$ & 90.320 & 90.329 & 90.338 & 90.343 & 90.386 & 90.340 & 90.172 & 89.814 & 89.097 & 88.569 & (16) \\
\hline$\delta^{\prime}\left({ }^{\circ}\right)$ & 1.0000 & 0.9932 & 0.9863 & 0.9814 & 0.9060 & 0.8176 & 0.7088 & 0.5631 & 0.3176 & 0.0000 & (22) \\
\hline$L(\AA)$ & 2.46782 & 2.46804 & 2.46825 & 2.46841 & 2.47077 & 2.47350 & 2.47675 & 2.48083 & 2.48658 & 2.49095 & $(A 1.2)$ \\
\hline$\Delta\left(^{\circ}\right)$ & 5.62656 & 5.60521 & 5.58350 & 5.56808 & 5.32508 & 5.02677 & 4.64175 & 4.09670 & 3.11159 & 1.73590 & $(A 1.2)$ \\
\hline$a_{\mathrm{PC}}(\AA)$ & 1.87272 & 1.87266 & 1.87260 & 1.87255 & 1.87174 & 1.87057 & 1.86888 & 1.86640 & 1.86239 & 1.85951 & $(A 1.2)$ \\
\hline$b_{\mathrm{PC}}(\AA)$ & 1.82490 & 1.82482 & 1.82475 & 1.82469 & 1.82386 & 1.82291 & 1.82178 & 1.82036 & 1.81821 & 1.81594 & $(A 1.2)$ \\
\hline$c_{\mathrm{PC}}(\AA)$ & 1.87031 & 1.86990 & 1.86949 & 1.86920 & 1.86502 & 1.86076 & 1.85631 & 1.85142 & 1.84475 & 1.83589 & $(A 1.2)$ \\
\hline$a(\AA)$ & 4.9119 & 4.9125 & 4.9131 & 4.9135 & 4.9202 & 4.9280 & 4.9373 & 4.9490 & 4.9658 & 4.9796 & (4) \\
\hline$x_{\mathrm{Si}}$ & 0.4716 & 0.4717 & 0.4718 & 0.4719 & 0.4731 & 0.4746 & 0.4766 & 0.4793 & 0.4843 & 0.4913 & (5) \\
\hline$x_{\mathrm{O}}$ & 0.4125 & 0.4125 & 0.4126 & 0.4126 & 0.4131 & 0.4136 & 0.4143 & 0.4152 & 0.4166 & 0.4177 & (6) \\
\hline$y_{\mathrm{O}}$ & 0.2655 & 0.2652 & 0.2650 & 0.2649 & 0.2625 & 0.2600 & 0.2572 & 0.2537 & 0.2481 & 0.2410 & (7) \\
\hline$z_{\mathrm{O}}$ & 0.7869 & 0.7871 & 0.7872 & 0.7874 & 0.7891 & 0.7913 & 0.7941 & 0.7981 & 0.8049 & 0.8123 & (9) \\
\hline$c(\AA)$ & 5.4035 & 5.4039 & 5.4044 & 5.4046 & 5.4090 & 5.4137 & 5.4192 & 5.4265 & 5.4383 & 5.4444 & (10) \\
\hline r.m.s.d. (\%) & 0.0003 & 0.0002 & 0.0003 & 0.0003 & 0.0007 & 0.0002 & 0.0008 & 0.0003 & 0.0009 & 0.0011 & \\
\hline
\end{tabular}

tures of $100 \mathrm{~K}$ has been chosen for temperatures within the range given by Antao (2016). Table 3 should be read from the top downwards. The first step is to calculate the Grimm and Dorner order parameter, $\delta$, via equation (1). Thereafter parameters $\phi_{\mathrm{v}}$ and $\phi_{\mathrm{h}}$ are calculated via equation $(A 1.1)$ and the fitting coefficients of Table 1 . In Table 3 , the equations used to calculate [Si+PC]-parameters from $\alpha_{\mathrm{PC}}$ down to $c_{\mathrm{PC}}$ are listed in the right-hand column. Thereafter the equations used to calculate the crystallographic parameters by reverse transformation from $[\mathrm{Si}+\mathrm{PC}]$-parameters are quoted in this column.

Calculated crystal structural parameters at the ten temperatures chosen are quoted below the horizontal rule in Table 3. The final three parameters, $y_{\mathrm{O}}, z_{\mathrm{O}}$ and $c$, were calculated via an iterative process using the GRG algorithm within the Microsoft Excel Solver software environment. Selfconsistent solutions to equations (7), (9) and (10) were sought, using trial values for these three parameters. Their values were refined in order to bring values of $b_{\mathrm{PC}}, c_{\mathrm{PC}}$ and $\alpha_{\mathrm{PC}}$ calculated from these equations into agreement with the values calculated from the coefficients relating to equation (A1.2) and quoted in Table 3. An indication of the self-consistency of the method is provided by the values of r.m.s. deviation quoted in the final line of Table 3. This parameter is defined in equation (23).

$$
\begin{aligned}
\text { r.m.s.d. }(\%)= & \left\{\left(\left[\frac{b_{\mathrm{PC}}(7)-b_{\mathrm{PC}}(A 1.2)}{b_{\mathrm{PC}}(A 1.2)}\right]^{2}\right.\right. \\
& +\left[\frac{c_{\mathrm{PC}}(9)-c_{\mathrm{PC}}(A 1.2)}{c_{\mathrm{PC}}(A 1.2)}\right]^{2} \\
& \left.\left.+\left[\frac{\alpha_{\mathrm{PC}}(10)-\alpha_{\mathrm{PC}}(A 1.2]}{\alpha_{\mathrm{PC}}(A 1.2)}\right]^{2}\right)^{1 / 2}\right\} \times 100 \%
\end{aligned}
$$

The numbers in the smallest brackets in equation (23) are equation numbers.

\subsection{Tilt angles and $[\mathrm{Ge}+\mathrm{PC}]$-parameters for $\mathrm{GeO}_{2}$}

Although the 16 structures of $\mathrm{GeO}_{2}$ refer to temperatures between 294 and $1344 \mathrm{~K}$ (Haines et al., 2002), the $\alpha$-quartztype structure for $\mathrm{GeO}_{2}$ is metastable with respect to a rutiletype phase at temperatures up to $\sim 1273 \mathrm{~K}$. It is the equilibrium phase only at higher temperatures up to the melting point of $\sim 1390 \mathrm{~K}$ (Liu \& Bassett, 1986). Landau parameters $T_{\mathrm{c}}$ and $T_{0}$ as for $\alpha$-quartz cannot be derived from structural data, as melting takes place on rising temperature before any such $\alpha \rightarrow \beta$ phase transition.

Length-based parameters $L, a_{\mathrm{PC}}, b_{\mathrm{PC}}, c_{\mathrm{PC}}$ are larger for $\mathrm{GeO}_{2}$ than for $\alpha$-quartz. Values of pseudocubic angle $\alpha_{\mathrm{PC}}$ are also uniformly larger, lying in the range $91.11^{\circ} \leq \alpha_{\mathrm{PC}} \leq 91.60^{\circ}$, compared to $88.53^{\circ} \leq \alpha_{\mathrm{PC}} \leq 90.45^{\circ}$ for $\alpha$-quartz. This observation signifies a greater degree of angular distortion of the tetrahedra. Larger values of $\lambda_{\mathrm{PC}}$ also point to tetrahedra that are comparatively more distorted, as discussed further in $\S 4.1$. Values of the parameter $3 V_{\text {tetra }} / V_{\mathrm{UC}}$ are higher for $\mathrm{GeO}_{2}$, this implying larger tilt angles: the greater the degree of tetrahedral tilting, the larger the proportion of space occupied by the tetrahedra. Tilt angles $\phi_{\mathrm{v}}$ and $\phi_{\mathrm{h}}$ are indeed consistently larger than for $\alpha$-quartz, although they span narrower ranges: $22.36^{\circ} \leq \phi_{\mathrm{v}} \leq 25.46^{\circ} ; 23.73^{\circ} \leq \phi_{\mathrm{h}} \leq 26.63^{\circ}$. As for $\alpha$-quartz, the smallest values in each range apply to the highest temperature. The implication is that $\mathrm{GeO}_{2}$ at $1344 \mathrm{~K}$ is still far away from an $\alpha \rightarrow \beta$ phase transition.

The ability to measure tilt angles directly in this work was exploited by adopting mean tilt angle as the order parameter $\delta$ for $\mathrm{GeO}_{2}$ instead of an equation of the form of (1). A quadratic function was fitted to the experimental data for this purpose, as summarized in equation (24).

$$
\delta(\mathrm{K})=a_{0}+a_{1}[T(\mathrm{~K})]+a_{2}[T(\mathrm{~K})]^{2} \quad[294<T(\mathrm{~K})<1344]
$$


Table 4

Fitting coefficients for parameters $L, \Delta, a_{\mathrm{PC}}, b_{\mathrm{PC}}$ and $c_{\mathrm{PC}}$ in $\mathrm{GeO}_{2}$ [equation (A1.2)].

\begin{tabular}{llrrr}
\hline Parameter & $a_{0}$ & $a_{1}$ & \multicolumn{1}{c}{$a_{2}$} & $a_{3}$ \\
\hline$L(\AA)$ & $2.56107 \times 10^{0}$ & $-2.07438 \times 10^{-2}$ & $-1.42516 \times 10^{-2}$ & $2.19897 \times 10^{-3}$ \\
$\Delta\left({ }^{\circ}\right)$ & $8.09667 \times 10^{0}$ & $1.44548 \times 10^{0}$ & $-7.63365 \times 10^{-1}$ & $8.11500 \times 10^{-1}$ \\
$a_{\mathrm{PC}}(\AA)$ & $2.03903 \times 10^{0}$ & $-5.80389 \times 10^{-2}$ & $3.20194 \times 10^{-2}$ & - \\
$b_{\mathrm{PC}}(\AA)$ & $1.91359 \times 10^{0}$ & $4.23398 \times 10^{-2}$ & $-3.62357 \times 10^{-2}$ & - \\
$c_{\mathrm{PC}}(\AA)$ & $2.04208 \times 10^{0}$ & $5.27147 \times 10^{-2}$ & $-1.94495 \times 10^{-2}$ & $8.98 \times 10^{-4}$ \\
\hline
\end{tabular}

The following coefficients and r.m.s. deviation apply: $a_{0}=$ $2.6242 \times 10^{1} ; a_{1}=-4.0000 \times 10^{-4} ; a_{2}=-1.5343 \times 10^{-6}$; r.m.s.d.: $0.14^{\circ}$. Just as the thermal Landau order parameter allowed lower-order polynomials to be fitted for $\alpha$-quartz, using the mean tilt angle here fulfils a similar purpose for the $\mathrm{GeO}_{2}$ fitting.

Values of parameters $L, \Delta, a_{\mathrm{PC}}, b_{\mathrm{PC}}, c_{\mathrm{PC}}$ and $\alpha_{\mathrm{PC}}$ from Table S2 for temperatures between 294 and $1344 \mathrm{~K}$ are plotted as points with associated error bars in Fig. 7.

For the curve-fitting in Fig. 7, the order parameter $\delta$ generated by equation (24) was adopted as the independent variable. The fitting coefficients listed in Table 4 relate to the reduced order parameter $\delta^{\prime}$ defined in equation (25).

$$
\delta^{\prime}=\frac{[\delta(T)-\delta(1344 \mathrm{~K})]}{[\delta(273 \mathrm{~K})-\delta(1344 \mathrm{~K})]}
$$

Thus $\delta^{\prime}=0$ at $1344 \mathrm{~K}$ and $\delta^{\prime}=1$ at $294 \mathrm{~K}$. The curve fitted for parameter $\alpha_{\mathrm{PC}}$ was calculated from equation (17), utilizing values for tilt angles $\phi_{\mathrm{v}}$ and $\phi_{\mathrm{h}}$ calculated from the coefficients in Table 5, using equation ( $A 1.1)$.

Whereas the curves for $L, \Delta$ and $c_{\mathrm{PC}}$ lie mostly within the bounds of the error bars of the experimental points, this does not apply to parameters $a_{\mathrm{PC}}, b_{\mathrm{PC}}$ and $\alpha_{\mathrm{PC}}$. It is further observed that successive experimental points for parameters

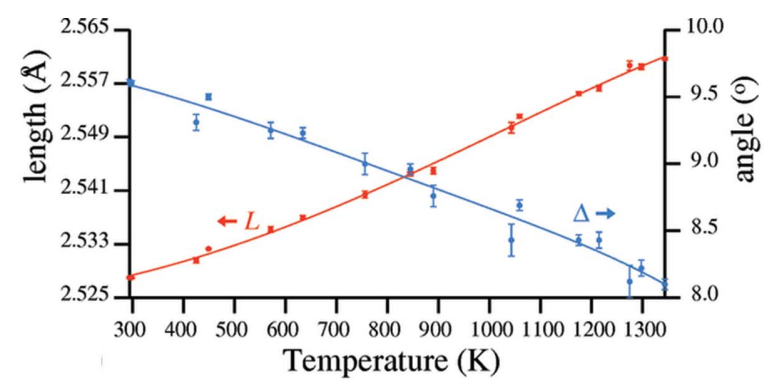

(a)

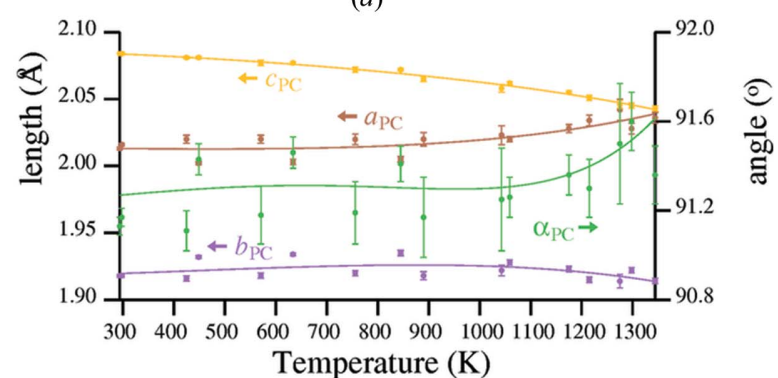

(b)

Figure 7

(a) Germanium framework parameters $L$ and $\Delta$; (b) oxygen pseudocube parameters $a_{\mathrm{PC}}, b_{\mathrm{PC}}, c_{\mathrm{PC}}$ and $\alpha_{\mathrm{PC}}$.
Table 5

Fitting coefficients for tilt angles $\phi_{\mathrm{v}}$ and $\phi_{\mathrm{h}}$ in $\mathrm{GeO}_{2}$.

\begin{tabular}{lcc}
\hline & \multicolumn{1}{c}{$\phi_{\mathrm{v}}\left(^{\circ}\right)$} & $\phi_{\mathrm{h}}\left(^{\circ}\right)$ \\
\hline$a_{0}$ & $-9.3270 \times 10^{2}$ & $-1.7978 \times 10^{2}$ \\
$a_{1}$ & $1.1504 \times 10^{2}$ & $2.4388 \times 10^{1}$ \\
$a_{2}$ & $-4.6455 \times 10^{0}$ & $-1.0021 \times 10^{0}$ \\
$a_{3}$ & $6.3008 \times 10^{-2}$ & $1.4212 \times 10^{-2}$ \\
r.m.s.d. $\left(^{\circ}\right)$ & $7.54 \times 10^{-2}$ & $1.44 \times 10^{-1}$ \\
\hline
\end{tabular}

$a_{\mathrm{PC}}$ and $b_{\mathrm{PC}}$ lie alternately above and below the fitted curves. At a given temperature, a point lying above the $a_{\mathrm{PC}}$ trendcurve corresponds to a point lying below the $b_{\mathrm{PC}}$ trend-curve, and vice versa. It transpires that points lying above the $a_{\mathrm{PC}}$ curve correspond to crystallographic data obtained from a sample measured with the Special Environment Powder Diffractometer at Argonne National Laboratory, whereas points lying below the curve relate to a different sample from the Polaris medium resolution diffractometer at the Rutherford Appleton Laboratory (Haines et al., 2002). In both cases, the Rietveld method was used in conjunction with time-offlight neutron powder diffraction data.

In view of the uncertainties in the values for parameters $a_{\mathrm{PC}}$, $b_{\mathrm{PC}}$ and $\alpha_{\mathrm{PC}}$, it was decided not to proceed with calculations of crystallographic parameters at interpolated temperatures, as carried out in Table 3 for $\alpha$-quartz. However, the separation of values for $a_{\mathrm{PC}}, b_{\mathrm{PC}}$ and $c_{\mathrm{PC}}$ into distinctive value-ranges is beyond question, this allowing a subsequent treatment of length-based tetrahedral distortion in $\$ 4$. Owing to the systematic variation with temperature of INA parameters $L, \Delta$ and $c_{\mathrm{PC}}$, it is reasonable to assume that the INA method is applicable, in principle, to $\mathrm{GeO}_{2}$ over the whole temperature range. The observed fluctuations in the other parameters correlate with two different samples and experimental stations.

\subsection{Curve-fitting and structural prediction for $\beta$-quartz}

The evolution with temperature of several derived parameters for $\alpha$ - and $\beta$-quartz is shown in Fig. 8, based on the structural refinements of Antao (2016). The unit-cell volume increases uniformly with temperature in the $\alpha$-phase and continues to rise beyond the phase transition to the $\beta$-phase to a maximum value at $921 \mathrm{~K}$, before falling back gently with increasing temperature (Antao, 2016). The volumes occupied by the $\mathrm{SiO}_{4}$ tetrahedra decrease strongly with temperature in the $\alpha$-phase, this being allowed by the decreasing mean tilt angle, and continue to fall gradually in the $\beta$-phase. The 

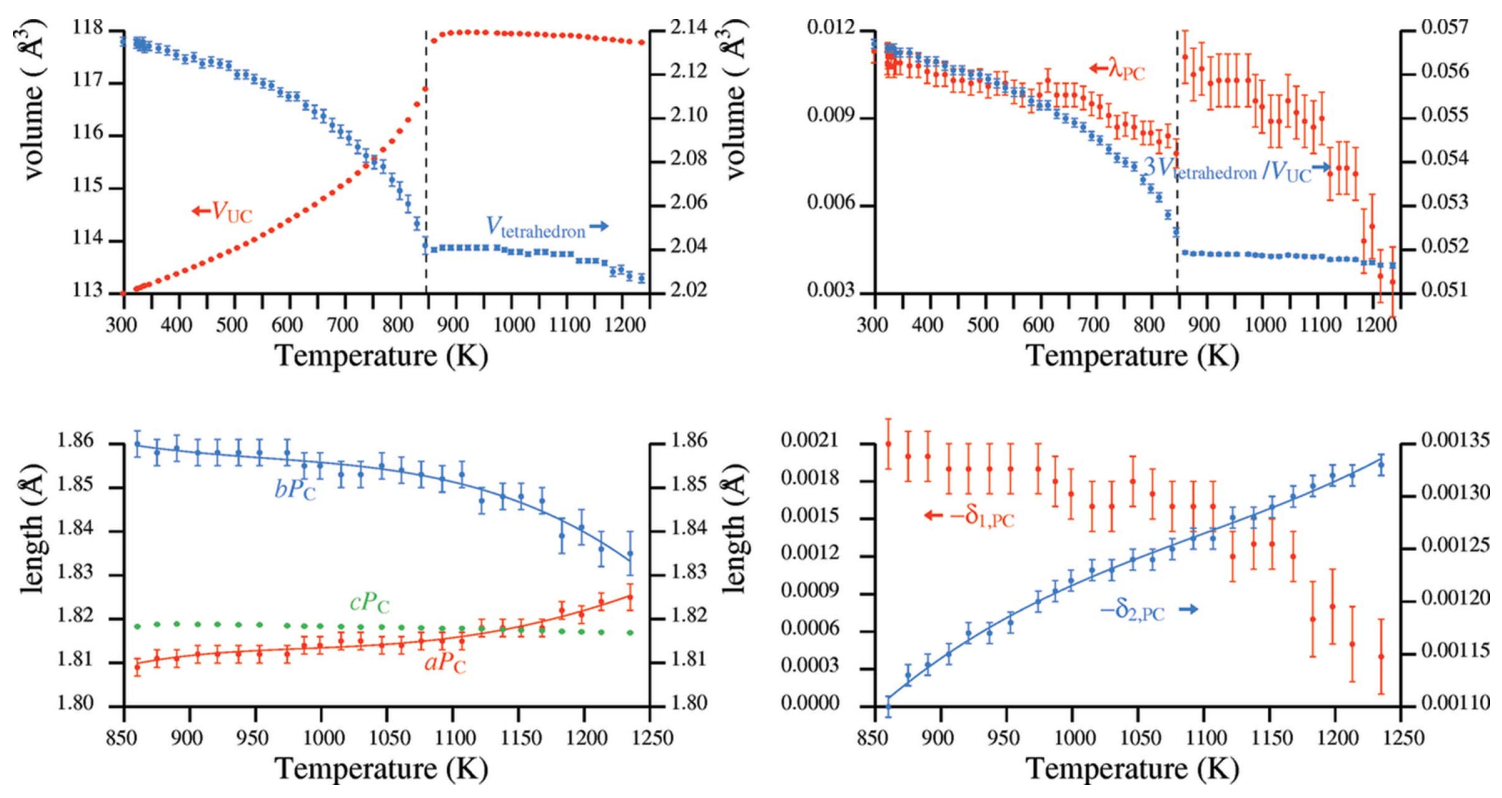

Figure 8

Parameter values for quartz derived from the data of Antao (2016): top over the whole temperature-range, bottom over the temperature range of $\beta$ quartz. $V_{\mathrm{UC}}$ : unit-cell volume; $V_{\text {tetrahedron }}: \mathrm{SiO}_{4}$-volume; $\lambda_{\mathrm{PC}}$ : length-based tetrahedral distortion; $a_{\mathrm{PC}}, b_{\mathrm{PC}}, c_{\mathrm{PC}}$ : pseudocubic parameters; $\delta_{1, \mathrm{PC}}, \delta_{2, \mathrm{PC}}$.

Table 6

Structural parameters of $\beta$-quartz at four temperatures calculated from the three fitted curves in Fig. 8 and associated polynomial coefficients in Table $7 \dagger$.

\begin{tabular}{llllll}
\hline$T(\mathrm{~K})$ & 900 & 1000 & 1100 & 1200 & Equation \\
\hline$\delta^{\prime}$ & 0.1067 & 0.3733 & 0.6400 & 0.9067 & $(A 2.1)$ \\
$a_{\mathrm{PC}}(\AA)$ & 1.8117 & 1.8135 & 1.8158 & 1.8220 & $(A 1.2) ;$ Table 7 \\
$b_{\mathrm{PC}}(\AA)$ & 1.8582 & 1.8558 & 1.8513 & 0.001314 & $(A 1.2) ;$ Table 7 \\
$-\delta_{2, \mathrm{PC}}(\AA)$ & 0.001146 & 0.001215 & 0.001265 & 4.9956 & $(17)$ \\
$a(\AA)$ & 4.9961 & 4.9967 & 4.9963 & 0.2106 & $(18)$ \\
$x$ & 0.2094 & 0.2095 & 0.2098 & 5.4515 & $(19)$ \\
$c(\AA)$ & 5.4563 & 5.4553 & 5.4535 & 7 \\
\hline
\end{tabular}

$\dagger$ Values of $x$ are in keeping with International Tables for Crystallography (Hahn, 1995) and not the convention employed by Antao (2016).

length-based tetrahedral distortion, $\lambda_{\mathrm{PC}}$, decreases with temperature in both phases, with a jump in values observed at the phase transition. Values ultimately attained at high temperature in the $\beta$-phase are lower than in the $\alpha$-phase. Parameter $3 V_{\text {tetrahedron }} / V_{\mathrm{UC}}$, which represents the fraction of space occupied by the $\mathrm{SiO}_{4}$ tetrahedra, decreases much more strongly in the $\alpha$-than in the $\beta$-phase. In the former case, the decrease is facilitated by the reduction in mean tilt angle. In the latter, the decrease indicates the stronger relative decrease in tetrahedral volume compared to unit-cell volume.

Pseudocubic parameters $a_{\mathrm{PC}}$ and $b_{\mathrm{PC}}$ for $\beta$-quartz show a stronger temperature-dependence than $c_{\mathrm{PC}}$, with opposite trends observed for $a_{\mathrm{PC}}$ and $b_{\mathrm{PC}}$. Curves have been fitted to the variations for $a_{\mathrm{PC}}$ and $b_{\mathrm{PC}}$, since equations (17) and (18) yield, by reverse transformation, values of the $a$ cell parameter and the oxygen $x_{\mathrm{O}}$ parameter.

The two parameters $\delta_{1, \mathrm{PC}}$ and $\delta_{2, \mathrm{PC}}$ are independent indicators of the deviation from regularity of the tetrahedra in $\beta$ quartz. They are defined as follows, whereby $x_{\mathrm{C}}$ is a reference value equal to $\frac{1}{2}-\frac{(3)^{1 / 2}}{6} \approx 0.21132$ (see $\$ S 3.2$ of the supporting information).

$$
\begin{gathered}
\delta_{1, \mathrm{PC}}=x_{\mathrm{O}}-x_{\mathrm{C}} \\
\delta_{2, \mathrm{PC}}=\frac{(3)^{1 / 2}}{9} \frac{c}{a}-x_{\mathrm{C}}
\end{gathered}
$$

A perfectly regular tetrahedron would have both $\delta_{1, \mathrm{PC}}$ and $\delta_{2, \mathrm{PC}}$ equal to zero. The contrary motion of their negative values with increasing temperature in the fourth diagram of Fig. 8 indicates that perfect tetrahedral regularity is not attained in $\beta$-quartz.

The strong monotonic variation of $-\delta_{2}$, PC with temperature allows a curve-fitting from which values of unit-cell parameter $c$ can be derived. Taken together, parameters $a_{\mathrm{PC}}, b_{\mathrm{PC}}$ and $\delta_{2 \text {, PC }}$ with associated curves enable prediction of the structures of $\beta$-quartz at interpolated temperatures. This procedure is shown in Table 6 for temperatures between 900 and $1200 \mathrm{~K}$ in $100 \mathrm{~K}$ intervals. The calculation procedure, which uses the coefficients listed in Table 7, may be inferred by reading the table from the top downwards. 


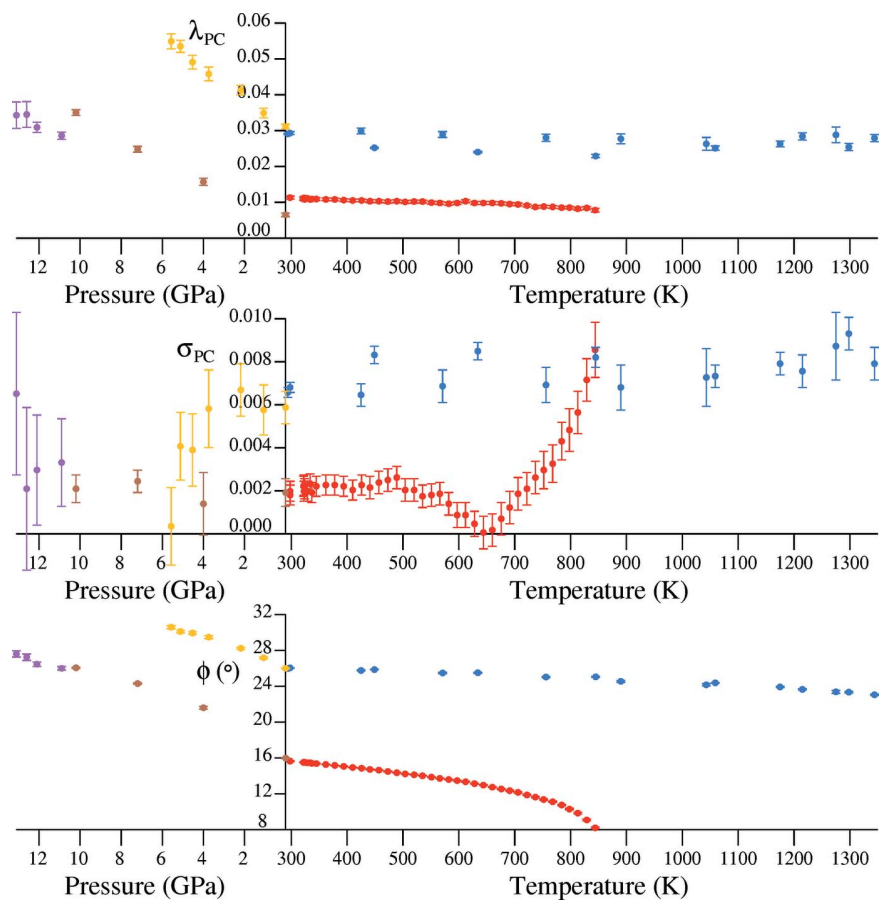

Figure 9

Comparison of tetrahedral distortions and tilt angles for $\alpha$-quartz and $\mathrm{GeO}_{2}$. Red points: $\alpha$-quartz at variable temperature (Antao, 2016); blue points: $\mathrm{GeO}_{2}$ at variable temperature (Haines et al., 2002); brown points: pressure-variation of $\alpha$-quartz up to $10.2 \mathrm{GPa}$ (Glinnemann et al., 1992) [The $r(+)$ setting according to Donnay \& Le Page (1978) in space group $P 3_{1} 21$ was used. An origin-shift of $\left[0,0, \frac{1}{3}\right]$ was applied in order to generate coordinates compatible with International Tables for Crystallography (Hahn, 1995)]; pink points: pressure variation of $\alpha$-quartz between 10.9 and 13.1 GPa (Kim-Zajonz et al., 1999) [The $r(+)$ setting according to Donnay \& Le Page (1978) in space group $P 3_{1} 21$ was used with coordinates compatible with International Tables for Crystallography (Hahn, 1995).]; yellow points: pressure variation of $\mathrm{GeO}_{2}$ up to $5.57 \mathrm{GPa}$ (Glinnemann et al. 1992).

\section{Discussion}

4.1. Comparison of the temperature- and pressure-evolution of quartz and $\mathrm{GeO}_{2}$ structures by means of tetrahedral distortion parameters

The length- and angle-based tetrahedral distortion parameters, $\lambda_{\mathrm{PC}}$ and $\sigma_{\mathrm{PC}}$, introduced by Reifenberg \& Thomas (2018) to enable a comparative overview of tetrahedral distortions under varying conditions of temperature and pressure, are plotted in Fig. 9 for $\alpha$-quartz and $\mathrm{GeO}_{2}$. The former corresponds to equation (21) and the latter parameter

$$
\sigma_{\mathrm{PC}}\left({ }^{\circ}\right)=\frac{\left|\alpha_{\mathrm{PC}}\left(^{\circ}\right)-90^{\circ}\right|+\left|\beta_{\mathrm{PC}}\left(^{\circ}\right)-90^{\circ}\right|+\left|\gamma_{\mathrm{PC}}\left({ }^{\circ}\right)-90^{\circ}\right|}{3}
$$

takes on the form of equation (28) when expressed in radians for $\alpha$-quartz or $\mathrm{GeO}_{2}$. These two parameters correspond to normal and shear distortions, respectively, and are normalized in order to reflect changes in shape and not volume.

$$
\sigma_{\mathrm{PC}}=\frac{\left|\alpha_{\mathrm{PC}}-(\pi / 2)\right|}{3}
$$

Also plotted are calculated values of mean tilt angle, $\phi$, in degrees.

It is observed that $\lambda_{\text {PC }}$ has uniformly higher values in $\mathrm{GeO}_{2}$ compared to $\alpha$-quartz at a given temperature or pressure, and further, that the application of hydrostatic pressure increases the length-based distortion in both crystal structures. The behaviour of $\sigma_{\mathrm{PC}}$ is more complicated. The red points for $\alpha$ quartz touch the $x$-axis at circa $640 \mathrm{~K}$, when $\alpha_{\mathrm{PC}}$ changes from values above $90^{\circ}$ to values below $90^{\circ}$ on increasing temperature. The blue points representing $\mathrm{GeO}_{2}$ are uniformly higher and show a weak dependence on temperature. By comparison, the application of pressures of up to $5.57 \mathrm{GPa}$ to $\mathrm{GeO}_{2}$ causes $\sigma_{\mathrm{PC}}$ to fall off, corresponding to a reduction in $\alpha_{\mathrm{PC}}$ from 91.0 to $89.9^{\circ}$. Such a fall-off is not observed for $\alpha$-quartz, with a small upwards trend in $\sigma_{\mathrm{PC}}$ seen. This results from $\alpha_{\mathrm{PC}}$ values that are consistently larger than $90^{\circ}$.

Although angular distortion $\sigma_{\mathrm{PC}}$ falls with increasing pressure in $\mathrm{GeO}_{2}$, this is not associated with the approach to a phase transition, as tilt angle $\phi$ takes on successively higher values with increasing pressure. This is the primary structural response of both $\mathrm{GeO}_{2}$ and $\alpha$-quartz to increasing pressure.

Taken together, these results support the view expressed by Glinnemann et al. (1992) that unpressurized $\mathrm{GeO}_{2}$ is a good model of the high-pressure structure of $\alpha$-quartz: the blue points for unpressurized $\mathrm{GeO}_{2}$ and the pink points for $\alpha$ quartz at high pressure occupy similar regions along the $y$-axis in the three diagrams of Fig. 9.

\subsection{The significance of tetrahedral distortion in quartz}

The term distortion implies deviation from an ideal. Two fundamental approaches are available for specifying such an ideal, the first referring to symmetry and the second to structure. The former leads naturally to considerations of group theory and the latter to crystal chemistry. In the case of quartz, as examined here, the aristotype corresponds to space group $\mathrm{P6}_{2} 22$ for $\beta$-quartz. On cooling below $846 \mathrm{~K}$, a displacive phase transition to its maximal sub-group $P 3_{2} 21$ takes place, this corresponding to $\alpha$-quartz. Bärnighausen (1980) has described this transition as lattice-equivalent (translationsgleich). The $\beta \rightarrow \alpha$ transition involves the loss of the twofold rotation symmetry in the parent space group along $\langle 210\rangle$ axes. It is therefore assigned the index 2 and notation $t 2$.

In terms of structure, the dominant feature observed in the lower symmetry, trigonal phase is tetrahedral tilting around the remaining $\langle 100\rangle$ twofold axes, along which the Si atoms lie. This twofold symmetry restricts the possible distortions of the $\mathrm{SiO}_{4}$ tetrahedra, such that the distortion of the $\mathrm{O}_{4}$ cages may be represented by pseudocubes in which two of the angles, $\beta_{\mathrm{PC}}$ and $\gamma_{\mathrm{PC}}$, are equal to $90^{\circ}$ (Fig. 2). A corollary is that four independent parameters are required to describe this distortion. The term pseudocube also implies the existence of an ideal of higher symmetry, i.e. the cube, which would be specified completely by one parameter, $a_{\mathrm{PC}}$, since the following three constraints apply: (i) $b_{\mathrm{PC}}=a_{\mathrm{PC}}$; (ii) $c_{\mathrm{PC}}=a_{\mathrm{PC}}$; (iii) $\alpha_{\mathrm{PC}}=90^{\circ}$. Such a cube corresponds to a perfectly regular $\mathrm{O}_{4}$ tetrahedron. 


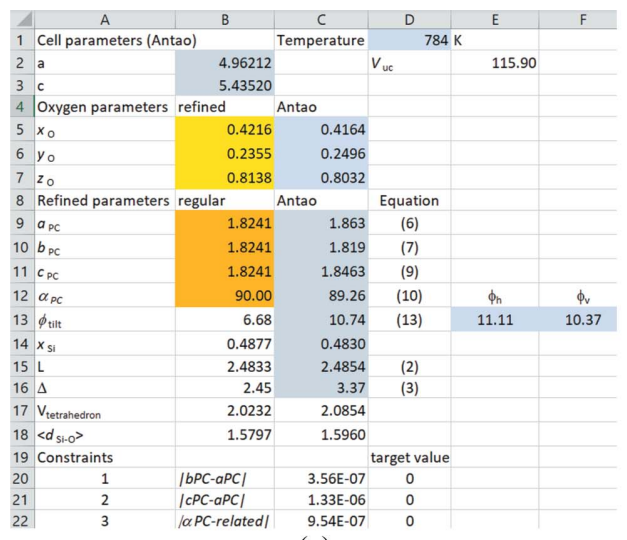

(a)

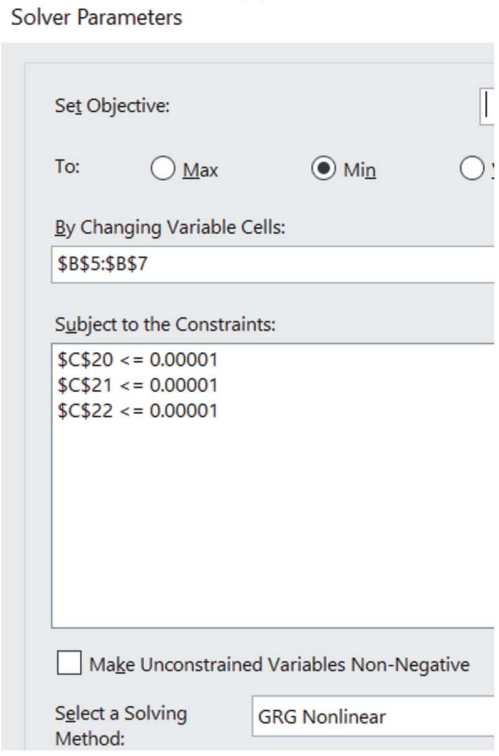

(b)

Figure 10

Procedure for calculating the oxygen positional parameters $\left(x_{\mathrm{O}}, y_{\mathrm{O}}, z_{\mathrm{O}}\right)$ and silicon $x$-coordinate $\left(x_{\mathrm{Si}}\right)$ of $\alpha$-quartz with regular tetrahedra for fixed cell parameters $a$ and $c$. (a) EXCEL spreadsheet. Cells B9 to B11 correspond to equations (6), (7) and (9), respectively. The formula in cell B12 calculates the pseudocubic angle $a_{\mathrm{PC}}$ according to equation (10). (b) Settings of the Solver.
Although space group symmetry allows regular $\mathrm{SiO}_{4}$ tetrahedra to exist in both $\beta$ - and $\alpha$-quartz, this ideal is not observed experimentally. For $\beta$-quartz, a regular $\mathrm{O}_{4}$ tetrahedron would impose restrictions on both oxygen parameter $x_{\mathrm{O}}$ and $c / a$ ratio such that $\delta_{1, \mathrm{PC}}=\delta_{2, \mathrm{PC}}=0$ [see equations (26) and (27) and the fourth diagram of Fig. 8]. For $\alpha$-quartz, the possibility of the existence of perfectly regular tetrahedra has been addressed by Smith (1963), who showed that this would require the $c / a$ ratio to be less than $\left(\frac{3}{2}\right)\left[(3)^{1 / 2}-1\right]$. Equations $(6),(7),(9)$ and (10) of the current work allow an extension of Smith's analysis to examine the consequences of regular tetrahedra for tilt angle. The above three constraints to form a cube may be applied, together with a fourth constraint that the $\mathrm{Si}$ ion be located at the centre-of-coordinates of its $\mathrm{O}_{4}$-cage.

Since Smith's $c / a$ criterion is fulfilled only by the nineteen structural refinements of Antao (2016) at temperatures $T \geq$ $566 \mathrm{~K}$, one way to address this question is to take the values for $a$ and $c$ at these temperatures and to apply the four constraints in a Microsoft Excel spreadsheet supported by the iterative GRG refinement in the Solver. The spreadsheet used for an example structure at $784 \mathrm{~K}$ is shown in Fig. 10(a), with the Solver settings for constraints (i)-(iii) above shown in Fig. 10(b).

The values of cells B5-B7 are allowed to vary subject to the constraints that cells $\mathrm{C} 20-\mathrm{C} 22$ contain values less than 0.00001 at the end of the refinement. In this connection, cell $\mathrm{C} 22$ contains the difference of the two terms in the numerator of the argument to the arccos function in equation (10). This is zero for an $\alpha_{\mathrm{PC}}$ angle of $90^{\circ}$. At the end of the refinement, cells B9-B12 (with light brown background) contain the parameters of a perfect cube. Further, the underlying equations, based on space group symmetry, guarantee that a system of interconnected regular $\mathrm{SiO}_{4}$ tetrahedra applies. The resulting oxygen $x, y, z$ parameters necessary for this are given in cells B5-B7 (with yellow background). Significant differences are observed relative to the experimental parameters of Antao (2016) (cells C5-C7), to which irregular tetrahedra with pseudocubic parameters in cells C9-C12 apply. The value of $x_{\mathrm{Si}}$ in cell B14 is calculated by applying the fourth constraint

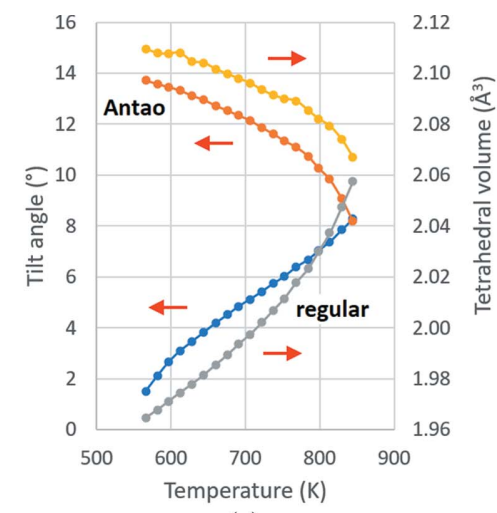

(a)

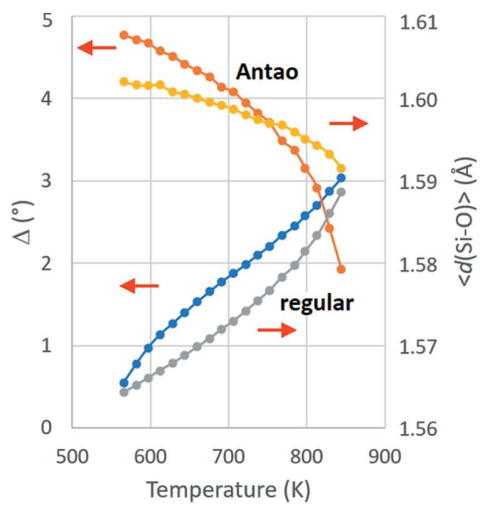

(b)

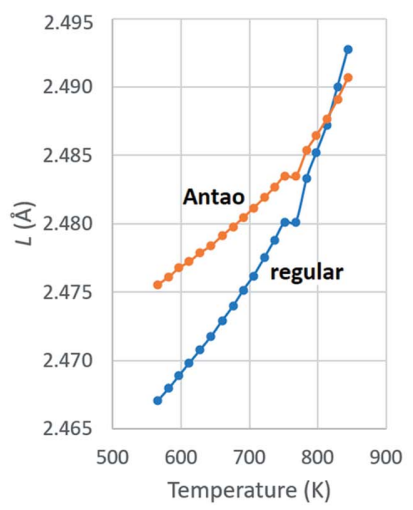

(c)

Figure 11

Comparison of structural variables for hypothetical $\alpha$-quartz structures containing regular $\mathrm{SiO}_{4}$ tetrahedra with the experimental structures of Antao (2016) at temperatures between 566 and $844 \mathrm{~K}$. (a) tilt angle $\phi$ and tetrahedral volume $V_{\text {tetra }} ;(b)$ mean $\mathrm{Si}-\mathrm{O}$ distance and Si-framework $\Delta$-parameter; (c) Si-framework $L$-parameter. 
Table 7

Fitting coefficients for parameters $a_{\mathrm{PC}}, b_{\mathrm{PC}}$ and $-\delta_{2, \mathrm{PC}}$ in $\beta$-quartz.

\begin{tabular}{|c|c|c|c|c|c|c|}
\hline$\underline{\text { Parameter }}$ & $a_{0}$ & $a_{1}$ & $a_{2}$ & $a_{3}$ & $a_{4}$ & r.m.s.d. \\
\hline$a_{\mathrm{PC}}(\AA)$ & $1.8099 \times 10^{0}$ & $2.1919 \times 10^{-2}$ & $-5.6548 \times 10^{-2}$ & $6.9719 \times 10^{-2}$ & $-1.9627 \times 10^{-2}$ & $8.19 \times 10^{-4}$ \\
\hline$b_{\mathrm{PC}}(\AA)$ & $1.8596 \times 10^{0}$ & $-1.6295 \times 10^{-2}$ & $3.0578 \times 10^{-2}$ & $-3.7656 \times 10^{-2}$ & $-3.0030 \times 10^{-3}$ & $1.41 \times 10^{-3}$ \\
\hline$-\delta_{2, \mathrm{PC}}(\AA)$ & $1.1080 \times 10^{-3}$ & $3.9126 \times 10^{-4}$ & $-3.4485 \times 10^{-4}$ & $1.8136 \times 10^{-4}$ & - & $4.81 \times 10^{-6}$ \\
\hline
\end{tabular}

relating to the location of the silicon ion at the centre-ofcoordinates of its $\mathrm{O}_{4}$ cage. The associated values of $L$ and $\Delta$, which relate to the Si-ion framework, are quoted in cells B15 and B16 by application of equations (2) and (3). Equation (13) is used to calculate the tilt angle, $\phi_{\text {tilt }}$, resulting for the structure with regular tetrahedra. $\alpha_{\mathrm{PC}}=90^{\circ}$ due to the regular tetrahedral geometry, so that $\phi_{\mathrm{v}}=\phi_{\mathrm{h}}$. This is is quoted in cell $\mathrm{B} 13$, whereby the value of $6.68^{\circ}$ is obtained for the $a$ and $c$ cell parameters of Antao (2016) at $784 \mathrm{~K}$. This differs significantly from the experimental value of $10.74^{\circ}$ (cell C13).

It is significant that regular tetrahedra give rise to tilt angles that increase from 1.50 to $8.27^{\circ}$ over the temperature range from 566 to $844 \mathrm{~K}$, whereas the distorted tetrahedra in the experimental structures of Antao (2016) have tilt angles that decrease from 13.72 to $8.19^{\circ}$ over this range [Fig. 11(a)]. That the primary Landau order parameter, i.e. tilt angle, should increase with increasing temperature is non-sensical. It follows that distorted tetrahedra in the $\alpha$-phase are necessary for Landau theory to be applicable. This situation is at variance with the behaviour of perovskites, i.e. systems of interconnected octahedra. In this context, the group-theoretical analysis of Howard \& Stokes (1998) found that, of the 15 possible sub-groups of cubic aristotype $P m \overline{3} m$ corresponding to different tilting patterns, only one was necessarily associated with octahedral distortion. They noted that such distortions were possible and expected in the other systems, but not required by geometry. These perovskite distortions have been analysed by other authors [see, for example, Thomas (1998); Tamazyan \& van Smaalen (2007)].

Fig. 11(a) also demonstrates the expected correlation between tilt angle and tetrahedral volume for both regular and distorted tetrahedra. The larger tetrahedral volumes of distorted tetrahedra correlate with larger mean $\mathrm{Si}-\mathrm{O}$ distances ${ }^{\mathbf{7}}, \mathbf{8}$ as well as angles $\Delta$ in the silicon ion framework [Fig. 11(b)]. The only case of parallel trends with temperature between regular and distorted tetrahedra relates to parameter $L$ in the silicon ion framework [Fig. 11(c)]. In general, the distorted tetrahedra in the Antao structures permit relatively longer $L$ values, leading to weaker $\mathrm{Si}$...Si repulsions.

Violation of the criterion due to Smith (1963) does not allow a network of regular tetrahedra to be formed for the cell parameters obtained at temperatures below $566 \mathrm{~K}$. His

\footnotetext{
${ }^{7}$ In a regular quartz, all four $\mathrm{Si}-\mathrm{O}$ distances are equal. In $\alpha$-quartz with distorted tetrahedra (Antao, 2016), there are two sets of two equal distances. ${ }^{8}$ The observed decrease in $\mathrm{Si}-\mathrm{O}$ distances with temperature in the experimental structures may an artefact arising from correlated thermal librations. Antao (2016) advocates a possible correction due to Downs et al. (1992).
}

Table 8

Pseudocubic parameters and tilt angles $\phi$ for the cell parameters of Antao (2016) at $345 \mathrm{~K}(a=4.91637 \AA ; c=5.40666 \AA)$ that result from four alternative sets of two applied constraints.

\begin{tabular}{lllll}
\hline \multicolumn{5}{l}{ Constraints applied } \\
\cline { 2 - 5 } Parameter & $\begin{array}{l}b_{\mathrm{PC}}=a_{\mathrm{PC}} \\
\alpha_{\mathrm{PC}}=90^{\circ}\end{array}$ & $\begin{array}{l}c_{\mathrm{PC}}=b_{\mathrm{PC}} \\
\alpha_{\mathrm{PC}}=90^{\circ}\end{array}$ & $\begin{array}{l}a_{\mathrm{PC}}=c_{\mathrm{PC}} \\
\alpha_{\mathrm{PC}}=90^{\circ}\end{array}$ & $\begin{array}{l}b_{\mathrm{PC}}=a_{\mathrm{PC}} \\
c_{\mathrm{PC}}=a_{\mathrm{PC}}\end{array}$ \\
\hline$a_{\mathrm{PC}}(\AA)$ & 1.8308 & 1.7948 & 1.8558 & 1.8083 \\
$b_{\mathrm{PC}}(\AA)$ & 1.8308 & 1.8474 & 1.8196 & 1.8083 \\
$c_{\mathrm{PC}}(\AA)$ & 1.8522 & 1.8474 & 1.8558 & 1.8083 \\
$\alpha_{\mathrm{PC}}\left({ }^{\circ}\right)$ & 90.00 & 90.00 & 90.00 & 92.40 \\
$\phi\left(^{\circ}\right)$ & 13.33 & 12.69 & 13.81 & 5.89 \\
\hline
\end{tabular}

limiting $c / a$-ratio of $\left(\frac{3}{2}\right)\left[(3)^{1 / 2}-1\right]$ corresponds to a tilt angle of zero. However, equations (6) to (10) allow an interconnected network provided that one of the constraints encoded in cells C20 to C22 of Fig. 10(a) is relaxed. The results yielded by the Microsoft Excel Solver for a representative structure at $345 \mathrm{~K}$ are given in Table 8 .

The parameters obtained are strongly dependent on the constraints applied. Tilt angle $\phi$ is highly variable and is to be compared with the experimental mean tilt angle at this temperature of $15.36^{\circ}$. Given this sensitivity, a further issue is to examine the pseudocubic parameter combinations that apply to all the experimental structures of Antao (2016). To this end, the polynomials in Table 2 for these parameters are plotted as a function of reduced Landau order parameter $\delta^{\prime}$ [as defined in equation (22)] in Fig. 12. Since it is not possible to adopt a regular tetrahedron (or equivalently perfect cube) as a reference over the whole temperature, another method of normalization has been adopted: values of $a_{\mathrm{PC}}, b_{\mathrm{PC}}$ and $c_{\mathrm{PC}}$ have been divided by the mean of the three values at each temperature. In the case of $\alpha_{\mathrm{PC}}$, absolute values have been divided by their median value $\left(89.48^{\circ}\right.$ ) over the whole temperature range. The corresponding normalized parameters, for which expansion/contraction effects have been factored out, are denoted by $a_{\mathrm{PC}}{ }^{\prime}, b_{\mathrm{PC}}{ }^{\prime}, c_{\mathrm{PC}}{ }^{\prime}$ and $\alpha_{\mathrm{PC}}{ }^{\prime}$.

The modes of distortion of the $\mathrm{O}_{4}$ tetrahedra vary over the temperature range investigated, with the extent of the variation in parameters increasing in the order $a_{\mathrm{PC}}{ }^{\prime}<b_{\mathrm{PC}}{ }^{\prime}<c_{\mathrm{PC}}{ }^{\prime}$. The values of parameters $a_{\mathrm{PC}}{ }^{\prime}$ and $c_{\mathrm{PC}}{ }^{\prime}$ approach each another as $\delta^{\prime} \rightarrow 1$. This behaviour is close to the third pair of constraints in Table 8 , for which the maximum tilt angle $\phi$ is observed. The unique increase in $c_{\mathrm{PC}}^{\prime}$ with increasing order parameter ( $\rightarrow$ tilt angle) may be rationalized by noting that increased tilt angles allow progressively larger values of $c_{\mathrm{PC}}$ to be accommodated for a given $c$ cell parameter. This analysis also allows an independent assessment of the validity of the 


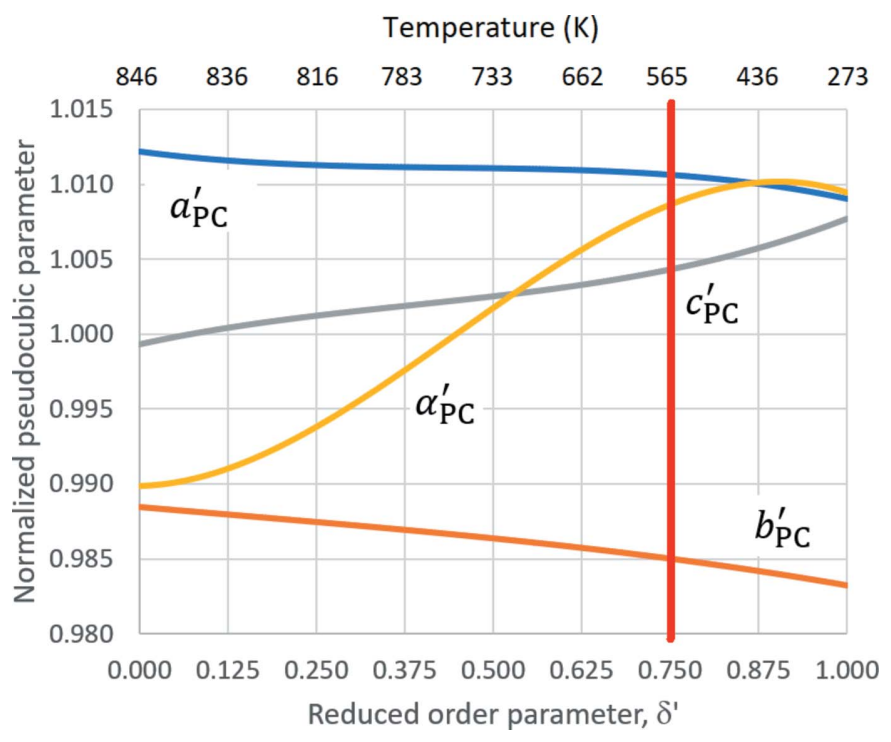

Figure 12

Variation of $a_{\mathrm{PC}}^{\prime}, b_{\mathrm{PC}}^{\prime}, c_{\mathrm{PC}}^{\prime}$ and $\alpha_{\mathrm{PC}}^{\prime}$ with reduced order parameter $\delta^{\prime}$ for $\alpha$ quartz. Temperatures corresponding to values of $\delta^{\prime}$ are indicated at the top of the graph. Regular tetrahedra are only possible at values of $\delta^{\prime}$ up to circa 0.75 , as denoted by the red vertical line.

rigid unit (phonon) mode (RUM) approximation, according to which displacive phase transitions in framework structures occur without any significant distortion of the $\mathrm{MO}_{4}$ tetrahedra (O'Keeffe \& Hyde, 1976; Giddy et al., 1993).

The ability to generate structural models for $\alpha$-quartz with alternative modes of tetrahedral distortion within the Microsoft Excel Solver, as shown in Fig. 10 and Table 8, is a useful by-product of the approach. Since this activity can be conducted independently of experimental diffraction data, it constitutes a simple, but versatile model-building method. It is likely to be useful for the modelling of auxetic (i.e. negative Poisson's ratio) or non-auxetic behaviour of $\alpha$-quartz subject to different constraints. Pioneering modelling work has been carried out here by Alderson \& Evans (2009), in which alternative combinations of tetrahedral rotation and dilation were examined.

Since the $[\mathrm{Si}+\mathrm{PC}]$ parameters can be reverse-transformed to crystallographic parameters, the method also allows the prediction of crystal structure at interpolated or extrapolated temperatures. This process, along with INA methods in general (Thomas, 2017; Reifenberg \& Thomas, 2018) will be of benefit when carrying out structural refinements of lower symmetry structures. The use of alternative, group-theoretical methods in this context was pioneered by Stokes \& Hatch (1988) and resulted in the ISOTROPY suite of programs (https://iso.byu.edu/iso/isotropy.php). In particular, the ISODISTORT web-based tool (Campbell et al., 2006), which acts as a gateway to the ISOTROPY suite, is geared towards analysing structural distortions. This proceeds by identifying the irreducible representations of parent space groups that are associated with distortions in their sub-groups. It would therefore be worthwhile to attempt a synthesis of the two approaches towards the quartz phase transition, both crystal chemical and group-theoretical.
It is not surprising that length- and angle-based parameters vary smoothly with temperature and pressure, since they fundamentally reflect the interactional potential energies and vibrational energies of the ions. This observation underlies the importance of crystallographic experiments carried out under variable $(p, T)$ conditions: they probe structural space. Furthermore, when lengths and angles are calculated, the complementary unit cell and atomic positional crystallographic parameters are combined in a Cartesian space that is conducive to establishing smooth trends with $(p, T)$. This is the essential purpose of the transformation from crystallographic to $[\mathrm{Si}+\mathrm{PC}]$ or, more generally, INA parameters. It therefore constitutes a technique that could become widely used in the refinement of structures examined under variable $(p, T)$ conditions.

It is intended to extend the current method to formulate more detailed structure-pieozelectric property relationships for single-crystal phosphates and arsenates $\left(\mathrm{ABO}_{4} ; \mathrm{A}=\right.$ B,Al,Ga,Fe; $B=$ P,As) (Baumgartner et al., 1984, 1989; Sowa, 1991, 1994; Nakae et al., 1995; Haines et al., 2004). These are homeotypic with $\alpha$-quartz and $\mathrm{GeO}_{2}$. However, the presence of two different cations leads to two symmetry-independent tetrahedra in the unit cell. For this reason, their structures have not been analysed here. However, continued application of the tilted regular tetrahedron model to these materials (Krempl, 2005) points to a need to discriminate more clearly between tetrahedral tilt and distortion in these materials.

The additional insight regarding tetrahedral distortions in quartz made possible by the data of Antao (2016) signals how high-quality crystallographic data can also contribute to a deeper understanding of phase transitions. This should act as a spur towards the more regular collection of crystallographic data of superior quality.

In seeking a microscopic interpretation of the Landau order parameter, attention in the literature has been focused until now on the tilt angle of the tetrahedra. This is indeed the dominant contribution. However, the inability of regular tetrahedra to generate appropriate values of tilt angle, as found here, demonstrates the importance of also taking tetrahedral distortion explicitly into consideration. Thus the comment of Taylor (1984), that (purely) 'tilting models of framework compounds fail to match the observed structural behaviour', has been addressed.

In general, the potential of crystal chemistry is far greater than merely offering a descriptive post-rationalization of experimentally determined structures. It is also able to offer a predictive framework for detailed dialogue with experiment.

\section{APPENDIX $A$}

A1. Curve-fitting coefficients for $\alpha$-quartz

A1.1. Tilt angles $\phi_{\mathrm{v}}, \phi_{\mathrm{h}}$ and $\phi_{\mathrm{m}}$. Polynomials of the form

$$
\phi=\sum_{i=0}^{n} a_{i} \delta^{i}
$$


were employed with $n=3$ and $\delta$ calculated from equation (1). Fitting coefficients $a_{i}$ are listed in Table 1.

A1.2. [Si+PC] parameters $L, \Delta, a_{P C}, b_{P C}$ and $C_{P C}$. Polynomials of the form of equation (A1.2) were employed with $n=3$.

$$
\phi=\sum_{i=0}^{n} \alpha_{i} \delta^{i}
$$

The reduced parameter applies here, as defined in equation (22). Fitting coefficients $a_{i}$ are listed in Table 2.

\section{A2. Curve-fitting coefficients for $\beta$-quartz}

Polynomials of the form of equation $(A 1.2)$ were employed with $n=4$ for parameters $a_{\mathrm{PC}}$ and $b_{\mathrm{PC}}$ and $n=3$ for parameter $-\delta_{2, \mathrm{PC}}$. The reduced parameter applies here, as defined in equation (A2.1), whereby the temperatures of $860 \mathrm{~K}$ and $1235 \mathrm{~K}$ correspond to the minimum and maximum temperatures of the structures reported by Antao (2016) for the $\beta$-phase. Fitting coefficients $a_{i}$ are listed in Table 7.

$$
\delta^{\prime} \frac{[\delta(T)-\delta(860 K)]}{[\delta(1235 K)-\delta(860 K)]}
$$

\section{Acknowledgements}

Open access funding enabled and organized by Projekt DEAL.

\section{References}

Alderson, A. \& Evans, K. E. (2009). J. Phys. Condens. Matter, 21, 025401.

Antao, S. M. (2016). Acta Cryst. B72, 249-262.

Bärnighausen, H. (1980). MATCH, 9, 139-175.

Baumgartner, O., Behmer, M. \& Preisinger, A. (1989). Z. Kristallogr. 187, 125-131.

Baumgartner, O., Preisinger, A., Krempl, P. W. \& Mang, H. (1984). Z. Kristallogr. 168, 83-91.

Campbell, B. J., Stokes, H. T., Tanner, D. E. \& Hatch, D. M. (2006). J. Appl. Cryst. 39, 607-614.
Carpenter, M. A., Salje, E. K. H., Graeme-Barber, A., Wruck, B., Dove, M. T. \& Knight, K. S. (1998). Am. Mineral. 83, 2-22.

Dolino, G. (1990). Phase Transit. 21, 59-72.

Donnay, J. D. H. \& Le Page, Y. (1978). Acta Cryst. A34, 584-594.

Downs, R. T., Gibbs, C. V., Bartelmehs, K. L. \& Boisen, M. B. Jr (1992). Am. Mineral. 77, 751-757.

Giddy, A. P., Dove, M. T., Pawley, G. S. \& Heine, V. (1993). Acta Cryst. A49, 697-703.

Glinnemann, J., King, H. E., Schulz, H., Hahn, T., Placa, S. J., La, \& Dacol, F. (1992). Z. Kristallogr. 198, 177-212.

Grimm, H. \& Dorner, B. (1975). J. Phys. Chem. Solids, 36, 407-413.

Hahn, T. (1995). Editor. International Tables for Crystallography, Vol. A, Space-Group Symmetry. Dordrecht: Kluwer.

Haines, J., Cambon, O., Astier, R., Fertey, P. \& Chateau, C. (2004). Z. Kristallogr. 219, 32-37.

Haines, J., Cambon, O. \& Hull, S. (2003). Z. Kristallogr. 218, 193-200.

Haines, J., Cambon, O., Philippot, E., Chapon, L. \& Hull, S. (2002). J. Solid State Chem. 166, 434-441.

Howard, C. J. \& Stokes, H. T. (1998). Acta Cryst. B54, 782-789.

Kihara, K. (1990). Eur. J. Mineral. 2, 63-78.

Kim-Zajonz, J., Werner, S. \& Schulz, H. (1999). Z. Kristallogr. 214, 324-330.

Krempl, P. W. (2005). J. Phys. IV Fr. 126, 95-100.

Liu, L. \& Bassett, W. A. (1986). Elements, Oxides, Silicates. HighPressure Phases with Implications for the Earth's Interior, p. 112. New York: Oxford University Press.

Megaw, H. D. (1973a). Crystal structures - a working approach, p. 268. London: Saunders.

Megaw, H. D. (1973b). Crystal structures - a working approach, pp. 453-456. London: Saunders.

Nakae, H., Kihara, K., Okuno, M. \& Hirano, S. (1995). Z. Kristallogr. 210, 746-753.

O'Keeffe, M. \& Hyde, B. G. (1976). Acta Cryst. B32, 2923-2936.

Reifenberg, M. \& Thomas, N. W. (2018). Acta Cryst. B74, 165-181.

Smith, G. S. (1963). Acta Cryst. 16, 542-545.

Sowa, H. (1991). Z. Kristallogr. 194, 291-304.

Sowa, H. (1994). Z. Kristallogr. 209, 954-960.

Stokes, H. T. \& Hatch, D. M. (1988). Isotropy Subgroups of the 230 Crystallographic Space Groups. Singapore: World Scientific.

Tamazyan, R. \& van Smaalen, S. (2007). Acta Cryst. B63, 190-200.

Taylor, D. (1984). Mineral. Mag. 48, 65-79.

Thomas, N. W. (1998). Acta Cryst. B54, 585-599.

Thomas, N. W. (2017). Acta Cryst. B73, 74-86. 